\title{
QUASICONFORMAL MAPPINGS AND CHORD-ARC CURVES
}

\author{
STEPHEN W. SEMMES
}

\begin{abstract}
Given a quasiconformal mapping $\rho$ on the plane, what conditions on its dilatation $\mu$ guarantee that $\rho(\mathbf{R})$ is rectifiable and $\left.\rho\right|_{\mathbf{R}}$ is locally absolutely continuous? We show in this paper that if $\mu$ satisfies certain quadratic Carleson measure conditions, with small norm, then $\rho(\mathbf{R})$ is a chord-arc curve with small constant, and $\rho(x)=\rho(0)+\int_{0}^{x} e^{a(t)} d t$ for $x \in \mathbf{R}$, with $a \in$ BMO having small norm. Conversely, given any such map from $\mathbf{R} \rightarrow \mathbf{C}$, we show that it has an extension to $\mathbf{C}$ with the right kind of dilatation. Similar results hold with $\mathbf{R}$ replaced by a chord-arc curve. Examples are given that show that it would be hard to improve these results. Applications are given to conformal welding and the theorem of Coifman and Meyer on the real analyticity of the Riemann mapping on the manifold of chord-arc curves.
\end{abstract}

Let $\rho$ be a quasiconformal map of the plane onto itself. Thus $\rho$ is a homeomorphism with locally integrable distributional derivatives, and $\rho_{\bar{z}}=\mu \rho_{z}$, where $\mu \in L^{\infty}(\mathbf{C}),\|\mu\|_{\infty}<1$. Here we use the notations

$$
f_{\bar{z}}=\bar{\partial} f=\frac{\partial}{\partial \bar{z}} f=\frac{1}{2}\left(\frac{\partial}{\partial x}+i \frac{\partial}{\partial y}\right) f, \quad f_{z}=\frac{1}{2}\left(\frac{\partial}{\partial x}-i \frac{\partial}{\partial y}\right) f .
$$

This function $\mu$ is called the complex dilatation of $\rho$. The mapping theorem for q.c. maps states that for each $\mu \in L^{\infty}(\mathbf{C}),\|\mu\|_{\infty}<1$, there is a q.c. map $\rho$ on $\mathbf{C}$ with dilatation $\mu$, and $\rho$ is unique up to normalization.

A basic problem is to understand how geometric properties of $\rho$ are reflected in $\mu$. For example, one would like to have natural conditions on $\mu$ which imply that $\rho(\mathbf{R})$ is rectifiable and $\left.\rho\right|_{\mathbf{R}}$ is absolutely continuous. This question arises naturally when considering problems in conformal mappings and conformal welding.

In this paper we obtain such estimates for the mapping theorem, and we also give some applications.

Our results involve $\mathrm{BMO}, A_{\infty}$ weights, chord-arc curves, and Carleson measures, and so we first review the appropriate definitions. A locally integrable function $f$ on $\mathbf{R}$ lies in BMO if

$$
\|f\|_{*}=\sup _{I} \frac{1}{|I|} \int_{I}\left|f(x)-f_{I}\right| d x
$$

Received by the editors June 1,1986 .

1980 Mathematics Subject Classification (1985 Revision). Primary 30G60; Secondary 42B20, $30 \mathrm{E} 20$.

Key words and phrases. Quasiconformal mapping, chord-arc curves, BMO, and Carleson measures. 
is finite, where $I$ is any interval, and where $f_{I}=|I|^{-1} \int_{I} f(y) d y$. The JohnNirenberg lemma states that there exist $C, \delta>0$ such that

$$
|I|^{-1} \int_{I} e^{\left|f(x)-f_{I}\right|} d x \leq C
$$

if $\|f\|_{*} \leq \delta$. See $[\mathbf{G}, \mathbf{J e}]$.

We can extend the definition of BMO to any locally rectifiable curve by replacing intervals with arcs. Thus any arclength parameterization preserves BMO.

Let $w(x)>0$ be locally integrable on $\mathbf{R}$. Set $w(E)=\int_{E} w(x) d x$, and let $|E|$ denote the Lebesque measure of $E$. We say that $w$ is an $A_{\infty}$ weight if for every $\varepsilon>0$ there is a $\delta>0$ such that if $I$ is any interval and $E \subseteq I$, then $|E| /|I|<\delta$ implies $w(E) / w(I)<\varepsilon$. See $[\mathbf{C F}, \mathbf{G}$, and $\mathbf{J e}]$ for basic properties of $A_{\infty}$ weights. An important fact is that $\log w \in \mathrm{BMO}$ if $w \in A_{\infty}$, and $\left\{\log w: w \in A_{\infty}\right\}$ spans an open subset of real-valued BMO, inducing a natural topology on $A_{\infty}$. In particular, there is a $\gamma>0$ so that $e^{b} \in A$ if $b$ is real valued and $\|b\|_{*} \leq \gamma$. The definition of $A_{\infty}$ can be extended to curves as before.

Suppose $h$ is an increasing homeomorphism of $\mathbf{R}$ onto itself, and define the operator $V_{h}$ by $V_{h} f=f \circ h$. Then $V_{h}$ determines a bounded operator on BMO if and only if $h$ is locally absolutely continuous and $h^{\prime} \in A_{\infty}$. (See [Js].) Results on $A_{\infty}$ imply that these homeomorphisms form a group.

When $n \geq 2$, the Jacobian of any q.c. $\operatorname{map} \rho$ on $\mathbf{R}^{n}$ is an $A_{\infty}$ weight, and $V_{\rho}$ preserves $\operatorname{BMO}\left(\mathbf{R}^{n}\right)$. If $n>2$ and $\rho$ also maps some hyperplane to itself, then the restriction of $\rho$ to that hyperplane is quasiconformal. When $n=2$, the restriction of $\rho$ to a line it preserves gives a homeomorphism of that line satisfying a doubling condition. Such a homeomorphism is often called quasisymmetric, and it generally is not even locally absolutely continuous. A homeomorphism $h$ of $\mathbf{R}$ onto itself that is locally absolutely continuous and satisfies $h^{\prime} \in A$ will be called strongly quasisymmetric.

Let $\Gamma$ be a locally rectifiable Jordan curve in the plane that passes through $\infty$, and let $z(t)$ be an arclength parameterization. We call $\Gamma$ a chord-arc curve with constant $k$ if $|s-t| \leq(1+k)|z(s)-z(t)|$ for all $s, t \in \mathbf{R}$. (That is, if the length of the chord is always at least $(1+k)^{-1}$ times the length of the arc.) Coifman and Meyer [CM2] proved that if $k$ is small enough then there is a real-valued $b \in \mathrm{BMO}(\mathbf{R})$ with small norm such that $z^{\prime}(t)=e^{i b(t)}$. Conversely, they showed that if $z^{\prime}(t)=e^{i b(t)}$ and $\|b\|_{*}$ is small enough, then $z(t)=\int_{0}^{t} e^{i b(s)} d s$ parameterizes a chord-arc curve with small constant. (Specifically, $\|b\|_{*} \approx \sqrt{k}$.)

More generally, if $z_{0}(\cdot)$ is an arclength parameterization of a fixed chord-arc curve $\Gamma_{0}$, then there is a $\delta_{0}>0$ so that if $b$ is real valued and $\|b\|_{*} \leq \delta_{0}$, then $\int_{0}^{t} e^{i b(s)} s_{0}^{\prime}(s) d s$ parameterizes a chord-arc curve. Moreover, David [D1] has shown that for chord-arc curves there is a natural choice of $\arg z^{\prime}$ lying in BMO that identifies the space of all chord-arc curves with an open subset of real-valued $\mathrm{BMO}(\mathbf{R})$. As with $A_{\infty}$, this allows us to think of the space of chord-arc curves as a topological space, in fact a Banach manifold.

Roughly speaking, $A_{\infty}$ weights are to chord-arc curves as $e^{x}$ is to $e^{i x}$.

Suppose $r(t)$ maps $\mathbf{R}$ homomorphically onto $\Gamma$. We say that $r(t)$ is a strongly quasisymmetric embedding if it is locally absolutely continuous, if $\Gamma$ is a chordarc curve, and if $\left|r^{\prime}(x)\right| \in A_{\infty}$. These conditions imply that $r$ is q.s. in the usual 
sense. A slight extension of Lemma 3 in [CM3] shows that if $r_{0}(t)$ is a strongly q.s. embedding then there is a $\delta_{0}>0$ such that if $b$ is a complex-valued BMO function and $\|b\|_{*} \leq \delta_{0}$, then

$$
r(t)=\int_{0}^{t} e^{b(s)} r_{0}^{1}(s) d s
$$

is still strongly q.s. One can extend the definition of strongly q.s. to mappings from one chord-arc curve to another.

A measure $\lambda$ on $\mathbf{C}$ is called a Carleson measure relative to a given chord-arc curve $\Gamma$ if there is a $C>0$ such that $|\lambda|\left(\left\{z:\left|z-z_{0}\right| \leq R\right\}\right) \leq C R$ for all $z_{0} \in \Gamma$ and $R>0$. The smallest such $C$ is the norm of $\lambda$.

Carleson measures are infamitely connected to BMO. By Fefferman-Stein, a function $f$ on $\mathbf{R}$ lies in BMO iff $|\nabla P f|^{2} y d x d y$ is a Carleson measure relative to $\mathbf{R}$, where $P f$ denotes the Poisson extension of $f$ to the upper half-plane (UHP). Varopoulos has shown that $f \in \operatorname{BMO}(\mathbf{R})$ iff $f$ has an extension $F$ to the upper half-plane such that $|\nabla F| d x d y$ is a Carleson measure.

Natural conditions on a dilatation for rectifiability results on the corresponding q.c. mapping turn out to be in terms of Carleson measures. Given a chord-arc curve $\Gamma$, let $\delta_{\Gamma}(z)=\operatorname{dist}(z, \Gamma)$. We define spaces $M(\Gamma)$ and $N(\Gamma)$ by

$M=M(\Gamma)=\left\{\mu \in L^{\infty}(\mathbf{C}):|\nabla \mu|^{2} \delta_{\Gamma}(z) d x d y\right.$ is a Carleson measure relative to $\left.\Gamma\right\}$ and

$N=N(\Gamma)=\left\{\mu \in L^{\infty}(\mathbf{C}):|\mu|^{2} \delta_{\Gamma}(z)^{-1} d x d y\right.$ is a Carleson measure relative to $\left.\Gamma\right\}$.

We define \|\|$_{M}$ and \|\|$_{N}$ to be the sum of $\|\mu\|_{\infty}$ and the square root of the Carleson norm. One should think of $N$ as being the stronger condition because it forces $\mu$ to vanish on $\Gamma$.

We shall also consider $M \cap N$ and $M+N=\left\{\mu \in L^{\infty}(\mathbf{C}): \mu=\mu_{1}+\mu_{2}, \mu_{1} \in M\right.$, $\left.\mu_{2} \in N\right\}$, with $\|\mu\|_{M+N}=\inf \left\{\left\|\mu_{1}\right\|_{M}+\left\|\mu_{2}\right\|_{N}: \mu=\mu_{1}+\mu_{2}\right\}$. One can easily characterize $M+N$ in terms of a Carleson measure condition on the $L^{2}$ mean oscillation of $\mu$ on the Whitney cubes of $\mathbf{C} \backslash \Gamma$.

THEOREM 0.1. Suppose $\Gamma_{0}$ is a chord-arc curve and $\mu \in M\left(\Gamma_{0}\right)+N\left(\Gamma_{0}\right)$, $\|\mu\|_{M+N} \leq \gamma_{0}=\gamma_{0}\left(\Gamma_{0}\right)$, where $\gamma_{0}>0$. Let $\rho: \mathbf{C} \rightarrow \mathbf{C}$ be a quasiconformal mapping with dilatation $\mu$. Then $\rho\left(\Gamma_{0}\right)$ is a chord-arc curve, $\left.\rho\right|_{\Gamma_{0}}$ is absolutely continuous, and there is a complex-valued function $a \in \mathrm{BMO}\left(\Gamma_{0}\right)$ with small norm, $\left.\|a\|_{*} \leq C\left(\Gamma_{0}\right)\|\mu\|_{M+N}\right)$, such that

$$
\rho\left(z_{1}\right)=\rho\left(z_{2}\right)+\int_{z_{2}}^{z_{1}} e^{a(w)} d w,
$$

where $z_{1}, z_{2} \in \Gamma_{0}$ and $\int_{z_{1}}^{z_{2}}$ denotes the integral along $\Gamma_{0}$ from $z_{2}$ to $z_{1}$. In particular, if $\Gamma_{0}=\mathbf{R}$, then the chord-arc constant of $\rho(\mathbf{R})$ is small.

This extends results of Carleson $[\mathbf{C}]$ and Dahlberg [Dh] for q.c. maps of the upper half-plane onto itself. In Dahlberg's case the dilatation satisfied condition $N$, while Carleson considered a stronger square-Dini condition.

It is surprising to me that these quadratic Carleson measure conditions $M$ and $N$ arise naturally in this context. Usually such a quadratic condition is accompanied by some cancellation, e.g., harmonicity, or one simply has an ordinary Carleson condition, as in Varopoulos' theorem.

The next result gives a partial converse to the above. 
THEOREM 0.2. Suppose $r(t)$ is a strongly quasisymmetric embedding of $\mathbf{R}$ into C. Then it has a quasiconformal extension $\rho: \mathbf{C} \rightarrow \mathbf{C}$ whose dilatation lies in $M \cap N$. In fact, for each $j \geq 0\left|y^{j} \nabla^{j} \mu\right|^{2}|y|^{-1} d x d y$ is a Carleson measure relative to $\mathbf{R}$.

We shall obtain estimates for $\nabla \rho$ too; see $\S 4$.

There is also a version of this when $\mathbf{R}$ is replaced by any given chord-arc curve $\Gamma_{0}$.

These two results do not characterize the q.c. maps $\rho: \mathbf{C} \rightarrow \mathbf{C}$ such that $\left.\rho\right|_{\mathbf{R}}$ is strongly q.s., even in the small constant case. One can easily construct q.c. maps that fix each point on $\mathbf{R}$ but whose dilatation satisfies nothing like $M$ or $N$. (Put a twist on each Whitney cube of $\mathbf{C} \backslash \mathbf{R}$, but without moving the boundary of the cube.)

Theorem 0.2 is not hard to get from a method of Tukia. Unfortunately, this relies on the Riemann mapping, and one would like to have something more explicit. In the case where $r(t)$ is a small perturbation of the identity (in the BMO topology), one can give an explicit formula, even when the starting-off curve is not $\mathbf{R}$. This formula is a variation of the Beurling-Ahlfors formula. (When the starting-off curve is not $R$, though, the formula is more complicated.) However, even the small perturbation case is interesting for applications. This is especially true since these applications also depend on Theorem 0.1 , which is available only in the small constant case.

For Theorem 0.1, the large constant case is extremely unclear. It is not even clear what the natural conjecture should be. In view of the example of [S1], the large constant case must be tricky, if tractable.

A natural way to attack Theorem 0.1 is to try to solve $(\bar{\partial}-\mu \partial) f=\mu_{z}$, where $f=\log \left(\rho_{z}\right)$. In particular, we want $\left\|\left.f\right|_{\Gamma_{0}}\right\|_{*}$ to be small. BMO estimates for $\bar{\partial}-\mu \partial$ are given in [S3], but $\mu_{z}$ is not the right kind of data. However, when $\mu$ lies in a variant of $M \cap N$, one can make this approach work, which we do in $\S 2$.

In general we have to take a different tack. The $\bar{\partial}-\mu \partial$ estimates in $[\mathbf{S 3}]$ show that if $\mu \in(M+N)\left(\Gamma_{0}\right)$ with small norm, then you can control the Cauchy integral on $\rho\left(\Gamma_{0}\right)$. From that you can show that $\rho\left(\Gamma_{0}\right)$ is rectifiable, chord-arc, and all the rest. This is done in $\S 3$.

This is reminiscent of [CM3], where certain mappings were estimated by seeing what they did to related operators, to wit, the Szëgo projection and the Cauchy integral. This is analogous to algebraic topology, where maps are studied by looking at what they do to attached algebraic structures, like homotopy and homology groups.

Although the method of $\S 3$ is more general, the approach in $\S 2$ is more direct and gives more information. For example, it allows you to also estimate $\nabla f=\nabla\left(\log \rho_{z}\right)$, and it tells you about the power series (in $\mu$ ). Also, in $\S 2$ there is a new estimate for $\bar{\partial}$ (Lemma 2.5) which is perhaps interesting in its own right.

In $\S 1$ we prepare for $\S \S 2$ and 3 by making certain useful reductions. $\S 4$ is devoted to Theorem 0.2 and its variants. We give applications in $\S \S 5$ and 6 to conformal welding and to the theorem of Coifman and Meyer on the analyticity of the Riemann mapping. 
A useful result that we use repeatedly in this paper is that for each chord-arc curve $\Gamma$ there is a bilipschitz map of $\mathbf{C}$ onto itself that takes $\mathbf{R}$ to $\Gamma$. See [Tu1, 2, JK].

The author is grateful to R. R. Coifman and P. W. Jones for helpful comments and suggestions, and also to the National Science Foundation for partial financial support in the form of a postdoctoral fellowship.

Some of the results of this paper were announced in the survey paper [S2]. The reader may find that paper useful for background information and for getting a better view of the "big picture".

1. Preliminary reductions for Theorem 0.1 . In this section we give a way to identify exponentials of small BMO functions, and then we show how this allows us to make a priori assumptions on $\mu$.

LEMMA 1.1. There is a small number $\delta_{0}$ with the following property. Let $v$ be any complex-valued function on $\mathbf{R}$, not identically 0 a.e. on any interval. Suppose also that there is a $\delta \leq \delta_{0}$ such that for each interval $I$ there is a constant $c_{I}$ so that

$$
|I|^{-1} \int_{I}\left|v(x)-c_{I}\right| d x \leq \delta|I|^{-1} \int_{I}|v(x)| d x .
$$

Then $v=e^{a}$, where $a \in \mathrm{BMO},\|a\|_{*} \leq C \delta$.

The converse is also true, with $c_{I}=\exp \left(|I|^{-1} \int_{I} a\right)$. This follows from JohnNirenberg and $\left|e^{x}-1\right| \leq|x| e^{|x|}$.

Observe that $\left|c_{I}\right|$ must be comparable to $|I|^{-1} \int_{I}|v|$, in fact,

$$
\left(1-\delta_{0}\right)\left|c_{I}\right| \leq|I|^{-1} \int_{I}|v| \leq\left(1+\delta_{0}\right)\left|c_{I}\right|
$$

Also, by hypothesis, $\int_{I}|v|$ is never 0 .

Let us first assume that $v$ is nonnegative. Let $a(x)=\log v(x)$ and $\beta_{I}=\log c_{I}$. From (1.2) and (1.3) we get

$$
|I|^{-1} \int_{I}\left|e^{a(x)-\beta_{I}}-1\right| d x \leq\left(1+\delta_{0}\right) \delta \leq 2 \delta .
$$

Because $\left|e^{x}-1\right| \geq \min \left(\frac{1}{2}|x|, .01\right)$,

$$
\left|e^{a(x)-\beta_{I}}-1\right| \geq 200 \delta \text { if }\left|a(x)-\beta_{I}\right|>400 \delta
$$

and if $\delta$ is sufficiently small. Hence (1.4) implies

$$
\left|\left\{x \in I:\left|a(x)-\beta_{I}\right|>400 \delta\right\}\right| \leq|I| / 100 .
$$

Therefore, $\|a\|_{*} \leq C \delta$, by Stromberg [Str] (see also [G, p. 270]). (This argument gives that $|a|<\infty$ a.e. in particular, so that $v>0$ a.e.)

Now suppose that $v$ is complex valued. The hypotheses of the lemma hold for $|v|$ with $c_{I}$ replaced by $\left|c_{I}\right|$, so that $\|\log |v|\|_{*} \leq C \delta$. If $u=v /|v|$, then

$$
u\left|c_{I}\right|-c_{I}=-u\left(|v|-\left|c_{I}\right|\right)+\left(v-c_{I}\right) \text {. }
$$

Thus

$$
|I|^{-1} \int_{I}|u(x)| c_{I}\left|-c_{I}\right| d x \leq 2 \delta|I|^{-1} \int_{I}|v(x)| d x
$$


whence $|I|^{-1} \int_{I}\left|u(x)-d_{I}\right| d x \leq 3 \delta$, where $d_{I}=c_{I}\left(\left|c_{I}\right|\right)^{-1}$. Altogether, we get that $u$ is unimodular and $\|u\|_{*} \leq 6 \delta$.

We need to find a good logarithm of $u$. This is equivalent to showing that if $z(t)$ is an arclength parameterization of a chord-arc curve $\Gamma$ with small constant, then $z^{\prime}(t)=e^{i b(t)}$, where $\|b\|_{*}$ is small. Indeed, the chord-arc constant $k$ of $\Gamma$ is small iff $\left\|z^{\prime}\right\|_{*}$ is, and $k \approx\left\|z^{\prime}\right\|_{*}^{2}$, by Lemma 6 in [CM2]. In that paper they show that such a $b$ does exist, and with the estimates we need for Lemma 1.1.

Let us indicate another proof of the existence of a good logarithm of $u$. Let $P u$ denote its Poisson integral in the UHP. Then $|P u| \leq 1$, and one can check that $|P u(z)| \geq 1-c\|u\|_{*} \geq 1-C \delta$ since $|u(x)| \equiv 1$. As in $[\mathbf{G}$, p. 348 and p. 372 , Exercise 11 ], there is a $C^{\infty}$ function $f(z)$ on the UHP such that $\|f-P u\|_{\infty} \leq\|u\|_{*}$ and $|\nabla f| d x d y$ is a Carleson measure with norm $\leq C\|U\|_{*}$. Thus $|f(z)| \geq 1-C \delta$ for all $z$, and because the UHP is simply connected, we can define $g(z)=\log f(z)$.

Thus $\nabla g=(\nabla f) f^{-1}$ defines a Carleson measure with norm $\leq C\|u\|_{*}$, and so by Varopoulos $[\mathbf{V}], g(z)$ has radial boundary values $g(x)$ a.e. on $\mathbf{R}, g \in \operatorname{BMO}(\mathbf{R})$, $\|g\|_{*} \leq C\|u\|_{*}$. Also, $\|f-P u\|_{\infty} \leq\|u\|_{*}$ implies that $\left\|P(u) f^{-1}-1\right\|_{\infty} \leq 2\|u\|_{*}$, and so if $\|u\|_{*} \leq 6 \delta \leq \frac{1}{2}$, we can define $\log \left(P(u) f^{-1}\right)$ so that its $L^{\infty}$ norm is $\leq 10\|u\|_{*}$. This also holds on $\mathbf{R}$, since $f$ has radial boundary values. Therefore we can write

$$
u(x)=e^{g(x)+\log \left(u(x) f(x)^{-1}\right)},
$$

and the exponent has BMO norm $\leq C\|u\|_{*}$.

This completes the proof of the lemma. Incidentally, once you know that $v=e^{a}$ where $\|a\|_{*}$ is small enough, then automatically $\delta \approx\|a\|_{*}$. This a priori estimate can be obtained from John-Nirenberg and $\left|e^{x}-1-x\right| \leq C|x|^{2} e^{|x|}$.

Lemma 1.1 also holds if $\mathbf{R}$ is replaced by any locally rectifiable curve, since you can pull back to $\mathbf{R}$ with an arclength parameterization.

LEMMA 1.5. To prove Theorem 0.1 , it suffices to prove the following. Let $\Gamma_{0}$ be any chord-arc curve, and let $z_{0}(\cdot)$ be an arclength parameterization. Then there is a small constant $\gamma_{0}=\gamma_{0}\left(\Gamma_{0}\right)>0$ so that if $\mu \in M\left(\Gamma_{0}\right)+N\left(\Gamma_{0}\right)$ has norm $\leq \gamma_{0}$, then $\rho \mid \Gamma_{0}$ is locally absolutely continuous and $v(x)=(d / d x)\left(\rho\left(z_{0}(x)\right)\right) z_{0}^{\prime}(x)^{-1}$ satisfies the hypotheses of Lemma 1.1 with $\delta \leq C\|\mu\|_{M+N}$.

If we define $\tilde{v}$ on $\Gamma_{0}$ by $\tilde{v}\left(z_{0}(x)\right)=v(x)$, then

$$
\rho\left(z_{2}\right)=\rho\left(z_{1}\right)+\int_{z_{1}}^{z_{2}} \tilde{v}(w) d w \quad \text { when } z_{1}, z_{2} \in \Gamma_{0} .
$$

Because of this, Lemma 1.5 follows from Lemma 1.1 and chasing definitions, except for showing that $\Gamma=\rho\left(\Gamma_{0}\right)$ is a chord-arc curve. In fact, if $\delta_{0}$ is small enough, and if $k_{0}$ and $k$ denote the chord-arc constants of $\Gamma_{0}$ and $\Gamma$, then $k \leq 1+k_{0}$. This follows from Lemma 3 in [CM3]. It is proved using a John-Nirenberg argument and the fact that $v=e^{a},\|a\|_{*}$ small.

LEMMA 1.6. To prove Theorem 0.1, it suffices to do the same thing as in Lemma 1.5, except that we may make the a priori assumption that $\mu$ is $C^{\infty}$ with compact support disjoint from $\Gamma_{0}$.

A well-known theorem states that if $\mu, \mu_{n} \in L^{\infty}(\mathbf{C}), \mu_{n} \rightarrow \mu$ a.e., $\sup \left\|\mu_{n}\right\|_{\infty}<$ 1 , if $\rho, \rho_{n}$ are q.c. maps with dilatations $\mu, \mu_{n}$, and if the $\rho$ 's are suitably normalized, 
then $\rho_{n} \rightarrow \rho$ uniformly on compact sets. To prove Lemma 1.6 we need to show that you can approximate $\mu$, and that everything is O.K. when $n \rightarrow \infty$. Let us first show how to approximate $\mu$.

LEMMA 1.7. If $\Gamma_{0}$ is a chord-arc curve, then we can find Lipschitz functions $m_{n}(z)$ such that $0 \leq m_{n}(z) \leq 1, m_{n}(z)$ has compact support disjoint from $\Gamma_{0}$, $m_{n}(z) \rightarrow 1$ as $n \rightarrow \infty$ if $z \notin \Gamma_{0}$, and $\left|\nabla m_{n}\right|^{2} \delta_{\Gamma_{0}}(z) d x d y$ is a Carleson measure with norm $\leq C\left(\Gamma_{0}\right)$.

When $\Gamma_{0}=\mathbf{R}$ this is straightforward. In general, we reduce to the real line using the fact that there is a bilipschitz map of $\mathbf{C}$ onto itself that takes $\mathbf{R}$ to $\Gamma_{0}$ [Tu1, 2; JK].

LEMMA 1.8. If $\mu \in M\left(\Gamma_{0}\right)+N\left(\Gamma_{0}\right)$, then there are $\mu_{n}$, smooth and with compact support disjoint from $\Gamma_{0}$, such that $\mu_{n} \rightarrow \mu$ a.e., $\left\|\mu_{n}\right\|_{\infty} \leq\|\mu\|_{\infty}$, and $\left\|\mu_{n}\right\|_{M+N} \leq C\|\mu\|_{M+N}$.

Consider $m_{n} \mu$. This has all the right properties except that it is not smooth. That is easily fixed.

Let $\rho_{n}, \rho: \mathbf{C} \rightarrow \mathbf{C}$ be q.c. maps with dilatations $\mu_{n}$ as in Lemma 1.7, suitably normalized so that $\rho_{n} \rightarrow \rho$ uniformly on compact sets. Let us show that if the conclusions of Lemma 1.5 hold for the $\rho_{n}$ 's and if $\|\mu\|_{M+N}$ is small, then they also hold for $\rho$.

By the remark after Lemma 1.5, each $\Gamma_{n}=\rho_{n}\left(\Gamma_{0}\right)$ has chord-arc constant $\leq$ $1+k_{0}$. Let $r_{n}(t)=\rho_{n}\left(z_{0}(t)\right)$ and $r(t)=\rho\left(z_{0}(t)\right)$, so that $r_{n}$ maps $\mathbf{R}$ to $\Gamma_{n}$ and is locally absolutely continuous. By the chord-arc condition,

$$
\begin{aligned}
& \int_{t_{1}}^{t_{2}}\left|r_{n}^{\prime}(t)\right| d t \leq\left(2+k_{0}\right)\left|r_{n}\left(t_{2}\right)-r_{n}\left(t_{1}\right)\right| . \\
& \text { (length of arc) } \quad \text { (length of chord) }
\end{aligned}
$$

Let $v_{n}(x)=(d / d x)\left(\rho_{n}\left(z_{0}(x)\right)\right) z_{0}^{\prime}(x)^{-1}$, which satisfies (1.2) with $\delta \leq C\|\mu\|_{M+N}$. By Lemma 1.1 and the fact that $\left|r_{n}^{\prime}(t)\right|=\left|v_{n}(t)\right|,\left\|\log \left|r_{n}^{\prime}(t)\right|\right\|_{*}$ is small, and so by the standard argument using John-Nirenberg, we have the reverse Schwartz inequality

$$
\left(|I|^{-1} \int_{I}\left|r_{n}^{\prime}\right|^{2}\right)^{1 / 2} \leq C\left(|I|^{-1} \int_{I}\left|r_{n}^{\prime}\right|\right)
$$

where $C$ does not depend on $n$ or on the interval $I$. From this, (1.9), and the uniform convergence on compact sets of the $\rho_{n}$, we conclude that $\left.\rho\right|_{\Gamma_{0}}$ is locally absolutely continuous, with derivative locally in $L^{2}$, and that $\Gamma=\rho\left(\Gamma_{0}\right)$ is a chord-arc curve with constant $\leq 1+k_{0}$.

It remains to show that $v(x)=(d / d x)\left(\rho\left(z_{0}(x)\right)\right) z_{0}^{\prime}(x)^{-1}$ also satisfies (1.2). Because $\left|z_{0}^{\prime}(x)\right| \equiv 1,(1.2)$ for $v_{n}$ is equivalent to

$$
|I|^{-1} \int_{I}\left|\frac{d}{d x}\left(\rho_{n}\left(z_{0}(x)\right)\right)-c_{I, n} z_{0}^{\prime}(x)\right| d x \leq \delta|I|^{-1} \int_{I}\left|\frac{d}{d x}\left(\rho_{n}\left(z_{0}(x)\right)\right)\right| d x
$$

where $I=\left[t_{1}, t_{2}\right]$ is any interval on $\mathbf{R}$ and $c_{I, n}$ is some constant.

Let us show that if we replace $c_{I, n}$ by

$$
c_{I, n}^{\prime}=\frac{\rho_{n}\left(z_{0}\left(t_{2}\right)\right)-\rho_{n}\left(z_{0}\left(t_{1}\right)\right)}{z_{0}\left(t_{2}\right)-z_{0}\left(t_{1}\right)},
$$


then (1.10) remains valid with $\delta$ replaced by $\left(1+k_{0}\right) \delta$. (This is helpful because we can control the limit of $c_{I, n}^{\prime}$.) To see this, observe that (1.10) implies

$$
\begin{aligned}
& |I|^{-1}\left|\left[\rho_{n}\left(z_{0}\left(t_{2}\right)\right)-\rho_{n}\left(z_{0}\left(t_{1}\right)\right)\right]-c_{I, n}\left[z_{0}\left(t_{2}\right)-z_{0}\left(t_{1}\right)\right]\right| \\
& \quad \leq \text { the left side of }(1.10) \leq \text { the right side of }(1.10) .
\end{aligned}
$$

Because $\left|z_{0}\left(t_{2}\right)-z_{0}\left(t_{1}\right)\right||I|^{-1} \geq\left(1+k_{0}\right)^{-1}$, we get that

$$
\left(1+k_{0}\right)^{-1}\left|c_{I, n}^{\prime}-c_{I, n}\right| \leq \text { the right side of (1.10). }
$$

Thus (1.10) still holds if $c_{I, n}$ is replaced by $c_{I, n}^{\prime}$ and $\delta$ is replaced by $\left(1+k_{0}\right) \delta$.

This together with the chord-arc condition (1.9) yield

$$
\begin{aligned}
& |I|^{-1} \int_{I}\left|\frac{d}{d x}\left(\rho_{n}\left(z_{0}(x)\right)\right)-c_{I, n}^{\prime} z_{0}^{\prime}(x)\right| d x \leq\left(1+k_{0}\right) \delta|I|^{-1} \int_{I}\left|\frac{d}{d x}\left(\rho_{n}\left(z_{0}(x)\right)\right)\right| d x \\
& \quad \leq\left(2+k_{0}\right)^{2} \delta|I|^{-1}\left|\rho_{n}\left(z_{0}\left(t_{2}\right)\right)-\rho_{n}\left(z_{0}\left(t_{1}\right)\right)\right| .
\end{aligned}
$$

Let us take the limit as $n \rightarrow \infty$. The right side tends to the correct thing, so that

$$
\begin{array}{r}
\limsup _{n \rightarrow \infty}|I|^{-1} \int_{I}\left|\frac{d}{d x} \rho_{n}\left(z_{0}(x)\right)-c_{I, n}^{\prime} z_{0}^{\prime}(x)\right| d x \\
\leq\left(2+k_{0}\right)^{2} \delta|I|^{-1}\left|\rho\left(z_{0}\left(t_{2}\right)\right)-\rho\left(z_{0}\left(t_{1}\right)\right)\right| \\
\leq\left(2+k_{0}\right)^{2} \delta|I|^{-1} \int_{I}\left|\frac{d}{d x}\left(\rho\left(z_{0}(x)\right)\right)\right| d x .
\end{array}
$$

The lim sup on the left is at least

$$
|I|^{-1} \int_{I}\left|\frac{d}{d x}\left(\rho\left(z_{0}(x)\right)\right)-c_{I}^{\prime} z_{0}^{\prime}(x)\right| d x .
$$

One way to see this is to approximate this last by

$$
|I|^{-1} \sum_{j=1}^{l}\left|\int_{I_{j}}\left(\frac{d}{d x} \rho\left(z_{0}(x)\right)-c_{I}^{\prime} z_{0}^{\prime}(x)\right) d x\right|,
$$

where $\left\{I_{j}\right\}$ is a finite partition of $I$. This can be approximated by

$$
|I|^{-1} \sum_{j=1}^{l}\left|\int_{I_{j}}\left(\frac{d}{d x}\left(\rho_{n}\left(z_{0}(x)\right)\right)-c_{I, n}^{\prime} z_{0}^{\prime}(x)\right) d x\right|
$$

because $\rho_{n} \rightarrow \rho$ locally uniformly. Clearly (1.13) is at most

$$
|I|^{-1} \int_{I}\left|\frac{d}{d x}\left(\rho_{n}\left(z_{0}(x)\right)\right)-c_{I, n}^{\prime} z_{0}^{\prime}(x)\right| d x .
$$

All these things together imply that (1.10) is valid with $\rho_{n}$ replaced by $\rho$ if we replace $\delta$ by $\left(2+k_{0}\right)^{2} \delta$.

This finishes the proof of Lemma 1.6.

2. Theorem 0.1 for nice $\mu$ 's by solving a Beltrami equation. A natural way to try to prove Theorem 0.1 is to try to get $\mathrm{BMO}\left(\Gamma_{0}\right)$ estimates for $\log \rho_{z}$ by solving the equation

$$
(\bar{\partial}-\mu \partial) \lambda=\mu_{z},
$$


where $\lambda=\log \rho_{z}$. (It is easy to check that (2.1) follows from $\rho_{\bar{z}}=\mu \rho_{z}$, by taking $\partial$ of both sides and dividing by $\rho_{z}$.) In [S3] related estimates for $\bar{\partial}-\mu \partial$ are obtained. For example, it is shown there that if $\mu \in M\left(\Gamma_{0}\right)+N\left(\Gamma_{0}\right)$ has small norm, and if $|a(z)| d x d y$ is a 2 -GCM relative to $\Gamma_{0}$ (which is just a little better than a Carleson measure), then you can solve $(\bar{\partial}-\mu \partial) f=a$ on $C$ with $\left.f\right|_{\Gamma_{0}} \in \operatorname{BMO}\left(\Gamma_{0}\right)$.

Unfortunately, in our situation $a=\mu_{z}$ is not a Carleson measure, but rather satisfies a quadratic Carleson measure condition if $\mu \in M$.

In general, when $\mu \in M+N$, it is not clear how to salvage this, and we have to do something else. (See §3.) However, if $\mu$ is slightly better than $M \cap N$, this argument can be made to work, which is what we do in this section.

The idea is as follows. Suppose $\mu \in M \cap N$, and let us try to solve (2.1): if $T=\partial \bar{\partial}^{-1}$,

$$
\begin{aligned}
\lambda & =(\bar{\partial}-\mu \partial)^{-1}\left(\mu_{z}\right)=\bar{\partial}^{-1}(I-\mu T)^{-1}\left(\mu_{z}\right) \\
& =\sum_{j=0}^{\infty} \bar{\partial}^{-1}(\mu T)^{j}\left(\mu_{z}\right)=\bar{\partial}^{-1} \mu_{z}+\sum_{j=1}^{\infty} \bar{\partial}^{-1}(\mu T)^{j-1}\left(\mu T \mu_{z}\right) \\
& =\bar{\partial}^{-1} \mu_{z}+(\bar{\partial}-\mu \partial)^{-1}\left(\mu T \mu_{z}\right) .
\end{aligned}
$$

Suppose we can show that $\left.\bar{\partial}^{-1}\left(\mu_{z}\right)\right|_{\Gamma_{0}} \in \mathrm{BMO}\left(\Gamma_{0}\right)$ and that $\left|T \mu_{z}\right|^{2} \delta_{\Gamma_{0}}(z) d x d y$ is a Carleson measure. Then $\left|\mu\left(T \mu_{z}\right)\right| d x d y$ is a Carleson measure, since $\mu \in N$ (and by Schwarz's inequality). We can then attack $(\bar{\partial}-\mu \partial)^{-1}\left(\mu T \mu_{z}\right)$ using [S3].

Let us make all this precise. First recall the definition of an $r$-GCM from [S3]. Let $a(z)$ and $0<\alpha<1$ be given. For $z \notin \Gamma$ define

$$
B_{z}=B_{z, \alpha}=\left\{w:|w-z|<\alpha \delta_{\Gamma}(z)\right\}
$$

and

$$
\tilde{a}(z)=\tilde{a}_{r, \alpha}(z)=\left(\left|B_{z}\right|^{-1} \int_{B_{z}}|a(w)|^{r} d u d v\right)^{1 / r} \quad(w=u+i v) .
$$

We say that $|a(z)| d x d y$ is an $r$-good Carleson measure, or $r$-GCM, relative to $\Gamma$ if $|\tilde{a}(z)| d x d y$ is a Carleson measure. This is independent of $\alpha, 0<\alpha<1$, different $\alpha$ yielding different norms. As $r$ increases the condition becomes stronger, and it coincides with the usual Carleson measure condition when $r=1$. An $r$-GCM is the same as a Carleson measure in the large, but locally it must have better integrability when $r>1$. This notion is needed for letting singular integrals (like $T)$ act on $a(z)$ which are unbounded on $L^{1}$ but are bounded on $L^{r}, 1<r<\infty$.

In this section we will work with $\mu$ 's that are a little better than $M \cap N$. Define 2-GMN $(\Gamma)$ to be the space of $\mu$ 's in $L^{\infty}(\mathbf{C})$ such that $|\mu|^{2} \delta_{\Gamma}(z)^{-1} d x d y$ and $|\nabla \mu|^{2} \delta_{\Gamma}(z) d x d y$ are 2-GCM's. The 2-GMN norm is defined in the obvious way. Similarly we define 2-GN $(\Gamma)=\left\{\mu \in L^{\infty}(\mathbf{C}):|\mu|^{2} \delta_{\Gamma}(z)^{-1} d x d y\right.$ is a 2-GCM $\}$. Thus 2 -GN $\subseteq N$ and 2 -GMN $\subseteq M \cap N$. Observe that if

$$
\left|\delta_{\Gamma}(z)^{j} \nabla^{j} \mu\right|^{2} \delta_{\Gamma}(z)^{-1} d x d y
$$

is a Carleson measure, $j=0,1,2$, then $\mu \in 2$-GMN, by Sobolev's lemma. 
PROPOSITION 2.4. There exists $\gamma_{0}=\gamma_{0}(\Gamma)>0$ such that the following holds. Suppose $\mu \in 2-\mathrm{GN}(\Gamma)$ has norm $\leq \gamma_{0}$ and $\nu \in 2-\mathrm{GMN}(\Gamma)$. Suppose also that $\mu, \nu$ are smooth and have compact support disjoint from $\Gamma$. Then there is a smooth function $\lambda$ such that $|\nabla \lambda| \in L^{2}(\mathbf{C}),(\bar{\partial}-\mu \partial) \lambda=\nu_{z}$ on $\mathbf{C}$, and $\left.\lambda\right|_{\Gamma} \in \operatorname{BMO}(\Gamma)$, with $\left\|\left.\lambda\right|_{\Gamma}\right\|_{*} \leq \mathbf{C}(\Gamma)\|\nu\|_{2-G M N}$. Also, $\nabla \lambda \in N(\Gamma),\|\nabla \lambda\|_{N} \leq \mathbf{C}(\Gamma)\|\nu\|_{2-G M N}$.

Because $\|\mu\|_{\infty}$ is small (and hence $<1$ ) and $\nu \in L^{2}(\mathbf{C})$,

$$
\lambda=(\bar{\partial}-\mu \partial)^{-1} \nu=\sum_{j=0}^{\infty} \bar{\partial}^{-1}(\mu T)^{j}(\nu)
$$

converges in the Sobolev space $L_{1}^{2}(\mathbf{C})=\left\{f: \nabla f \in L^{2}\right\}$ (since $T$ is unitary on $L^{2}$ ). Because $\mu$ and $\nu$ are smooth and compactly supported, this series can be differentiated arbitrarily often and it still converges in $L_{1}^{2}$, so that $\lambda$ is $C^{\infty}$.

Before proving the BMO estimates for $\lambda$ let us show how the proposition implies Theorem 0.1 when $\mu \in 2-\operatorname{GMN}\left(\Gamma_{0}\right)$ has small enough norm. Assume first that $\mu$ is also smooth and has compact support disjoint from $\Gamma_{0}$. Let $\lambda$ be as in the proposition with $\nu=\mu_{z}$. Since $\mu$ is compactly supported, $\lambda$ is holomorphic on $R<|z|<\infty$ for some $R$. From $\nabla \lambda \in L^{2}$ we get that $\lambda^{\prime}(z)=c / z^{2}+\cdots$ at $\infty$, so that $\lambda(z)=-c / z+\cdots$ at $\infty$, by adding a constant if necessary. Standard arguments (see [AB, especially the proof of Lemma 7], or [A2, p. 95]) show that one can find a q.c. map $\rho: \mathbf{C} \rightarrow \mathbf{C}$ with dilatation $\mu$ such that $\log \rho_{z}=\lambda$.

Let $z_{0}(t)$ be an arclength parameterization of $\Gamma_{0}$. Then

$$
\rho\left(z_{0}(s)\right)=\rho\left(z_{0}(0)\right)+\int_{0}^{s} \frac{d}{d s} \rho\left(z_{0}(s)\right) d s .
$$

By the chain rule,

$$
\begin{aligned}
\frac{d}{d s} \rho\left(z_{0}(s)\right) & =\rho_{z} z_{0}^{\prime}(s)+\rho_{\bar{z}} \overline{z_{0}^{\prime}(s)} \\
& =\rho_{z}\left(z_{0}(s)\right) z_{0}^{\prime}(s)\left(1+\mu\left(z_{0}(s)\right) \frac{\overline{z_{0}^{\prime}(s)}}{z_{0}^{\prime}(s)}\right) .
\end{aligned}
$$

Thus

$$
\rho\left(z_{0}(s)\right)=\rho\left(z_{0}(0)\right)+\int_{0}^{s} \rho_{z}\left(z_{0}(s)\right)(1+\alpha(s)) z_{0}^{\prime}(s) d s
$$

where $\|\alpha\|_{L^{\infty}(\mathbf{R})}$ is small. This and the smallness of $\left\|\left.\log \rho_{z}\right|_{\Gamma_{0}}\right\|_{*}$ imply the conclusion of Theorem 0.1 .

As in Lemma 1.6, we can get rid of the assumption that $\mu \in C^{\infty}$ with support disjoint from $\Gamma_{0}$. (Here one needs the analogue of Lemma 1.8 with $M+N$ replaced by 2-GMN, but that is easy.) Thus Proposition 2.4 implies that the conclusion of Theorem 0.1 holds if $\mu \in 2-\operatorname{GMN}\left(\Gamma_{0}\right)$ has small enough norm.

To prove Proposition 2.4 , we need an estimate for $\bar{\partial}$.

LEMMA 2.5. Suppose that $\nu$ is $C^{\infty}$, has compact support disjoint from $\Gamma_{0}$, and that $\left|\nu_{z}\right|^{2} \delta_{\Gamma}(z) d x d y$ and $|\nu|^{2} \delta_{\Gamma}(z)^{-1} d x d y$ are $2-G C M ' s$. Then $\left.\bar{\partial}^{-1}\left(\nu_{z}\right)\right|_{\Gamma}$ lies in $\mathrm{BMO}(\Gamma)$ and $\left|T\left(\nu_{z}\right)\right|^{2} \delta_{\Gamma}(z) d x d y$ is a 2 -GCM relative to $\Gamma$, with norms depending only on the above two 2-GCM norms. 
This should be compared with Wolff's $\bar{\partial}$ estimate [G, p. 322]. There the extra cancellation came from an estimate on the derivative of the data, while here it comes from an estimate on the primitive $\nu$ of the data $\nu_{z}$.

Let us derive Proposition 2.4 from the lemma. As in (2.2),

$$
\lambda=\bar{\partial}^{-1}\left(\nu_{z}\right)+(\bar{\partial}-\mu \partial)^{-1}\left(\mu T \nu_{z}\right)
$$

By the lemma the first piece is O.K. and $\mu\left(T \nu_{z}\right)$ is a 2 -GCM, since $\mu \in 2 \mathrm{GN}(\Gamma)$. Because $\|\mu\|_{N}$ is small, we can solve $(\bar{\partial}-\mu \partial)^{-1}\left(\mu T \nu_{z}\right)$ with the desired estimates by Theorem 5.2 in [S3]. (That theorem is stated in a complicated way to circumvent distributional technicalities. In our case the technicalities do not arise because of the a priori assumptions on $\mu$ and $\nu$.)

Let us prove the lemma. Set

$$
F(\varsigma)=\bar{\partial}^{-1}\left(\nu_{z}\right)(\varsigma)=\int_{\mathbf{C}} \nu_{z}(w) \frac{1}{\varsigma-w} d u d v
$$

(From now on we shall ignore inessential multiplicative constants.) Consider first $\left.F\right|_{\Gamma}$. Since $\operatorname{supp} \nu_{z} \cap \Gamma=\varnothing$, integration by parts gives

$$
F(\varsigma)=\int_{\mathbf{C}} \nu(w) \frac{1}{(\varsigma-w)^{2}} d u d v \quad \text { for } \varsigma \in \Gamma .
$$

Assume first that $\Gamma=\mathbf{R}$. To estimate $\left\|\left.F\right|_{\mathbf{R}}\right\|_{*}$ it is enough to pair $\left.F\right|_{\mathbf{R}}$ with any $g$ in (real-variable) $H^{1}$. By Fubini, we get

$$
\int_{\mathbf{R}} F g|d \zeta|=\int_{\mathbf{C}} \nu(w)\left(\int_{\mathbf{R}} \frac{1}{(\zeta-w)^{2}} g(\varsigma)|d \zeta|\right) d u d v \text {. }
$$

The inner integral is the derivative of the Cauchy integral of $g$, and hence has an integrable area function. Because $|\nu(z)|^{2}|y|^{-1} d x d y$ is a Carleson measure, (2.4) can be estimated as in Remark (a) on pp. 148-149 of [FS] or p. 313 of [CMS].

When $\Gamma$ is a general chord-arc curve, one argues similarly. One takes $g$ to be an arbitrary function in atomic $H^{1}(\Gamma)$, for which one can prove the area function estimates in the usual way from the $L^{2}$ case in $[\mathbf{J K}]$ and the boundedness of the Cauchy integral on all chord-arc curves ([D3]; see also [CDM]). The last step of the argument can be reduced to the line using a bilipschitz change of variables [Tu1, 2, JK].

We are left with estimating

$$
\partial F(\varsigma)=T\left(\nu_{z}\right)(\varsigma)=\int_{\mathbf{C}} \frac{1}{(\varsigma-w)^{2}} \nu_{z}(w) d u d v
$$

where the integral is interpreted as a principal value. We assume first that $\Gamma=\mathbf{R}$, $\operatorname{supp} \nu \subseteq \mathrm{UHP}$, and $\varsigma \in \mathrm{UHP}$. Our calculations will be similar to those in the proof of Proposition 4.1(a) in [Se3].

Let $x_{0} \in \mathbf{R}$ and $R>0$ be given. We want to show that $|\partial F(\varsigma)|^{2}|\eta| d \xi d \eta$ is a 2-GCM, $\varsigma=\xi \in i \eta$, and so we must estimate

$$
\begin{aligned}
& \iint_{\substack{\zeta \in U_{H P} \\
\left|\zeta-x_{0}\right| \leq R}}|H(\varsigma)| d \xi d \eta, \\
& \quad \text { where } H(\varsigma)=\left(\left|B_{\varsigma}\right|^{-1} \int_{B_{\varsigma}}\left(|\partial F(w)|^{2} v\right)^{2} d u d v\right)^{1 / 2}
\end{aligned}
$$


and where $B_{\varsigma}$ is as in (2.3) with $\alpha=.1$, say. We need to break up $\partial F$ into two pieces, near and far from the singularity in (2.7).

Let $\varphi(w)$ be a $C^{\infty}$ function supported in $|w|<1$ such that $\varphi(w)=1$ if $|w| \leq \frac{1}{2}$. Define $\varphi_{s}(w)=\varphi((w-\varsigma) / 10 \eta)$. Thus

$$
\begin{aligned}
\partial F(\varsigma) & =\int_{\mathbf{C}} \frac{1}{(\varsigma-w)^{2}} \varphi_{\varsigma}(w) \nu_{z}(w) d u d v+\int_{\mathbf{C}} \frac{1}{(\varsigma-w)^{2}}\left(1-\varphi_{\zeta}(w)\right) \nu_{z}(w) d u d v \\
& =G_{1}(\varsigma)+G_{2}(\varsigma) .
\end{aligned}
$$

To control the contribution of $G_{1}(\varsigma)$ we need that

$$
\begin{aligned}
& \left(\left|B_{\zeta}\right|^{-1} \int_{B_{\varsigma}}\left(\left|G_{1}(w)\right|^{2} v\right)^{2} d u d v\right)^{1 / 2} \\
& \quad \leq C\left(\left|B_{\zeta}\right|^{-1} \int_{\tilde{B}_{\varsigma}}\left(\left|v_{z}(w)\right|^{2} v\right)^{2} d u d v\right)^{1 / 2},
\end{aligned}
$$

where $\tilde{B}_{z}$ is the double of $B_{z}$. This is proved just like (4.7) in [Se 3]. (The point is that $T$ is bounded on $L^{4}$ and $G_{1}(\varsigma)$ only involves $\nu_{z}(w)$ for $w$ near $\left.\varsigma.\right)$ Using (2.9) the contribution of $G_{1}$ in the integral in (2.8) is controlled by the 2-GCM norm of $\left|v_{\boldsymbol{z}}(w)\right|^{2} v d u d v$.

For $G_{2}$ we integrate by parts to obtain

$$
G_{2}(\varsigma)=\int_{\mathbf{C}} \frac{2}{(\varsigma-w)^{3}}\left(1-\varphi_{\varsigma}(w)\right) \nu(w) d u d v+\int_{\mathbf{C}} \frac{1}{(\varsigma-w)^{2}} \partial \varphi_{\varsigma}(w) \nu(w) d u d v
$$

The second term is localized just like $G_{1}$, and it is handled in the same way, using that $|\nu(w)|^{2}|v|^{-1} d u d v$ is a 2-GCM. (This time it is easier, because the singularity is killed.)

This leaves the first term, which is dominated by

$$
S(\varsigma)=\int_{\mathrm{UHP}} \frac{1}{|\varsigma-\bar{w}|^{3}}|\nu(w)| d u d v
$$

because $|\varsigma-w|$ and $|\varsigma-\bar{w}|$ are comparable if $\varsigma, w \in$ UHP and $|\zeta-w| \geq \eta / 10$. Clearly $S(z) \leq C S(\varsigma)$ if $|z-\varsigma| \leq \eta / 10$, by looking at the kernel, and so

$$
\left(\left|B_{\varsigma}^{-1}\right| \int_{B_{\varsigma}}\left(|S(w)|^{2} v\right)^{2} d u d v\right)^{1 / 2} \leq C|S(\varsigma)|^{2} \eta
$$

Hence the contribution to the integral in (2.8) is at most

$$
\iint_{\substack{s \in \text { UHP } \\\left|\varsigma-x_{0}\right| \leq R}}|S(\varsigma)|^{2} \eta d \xi d \eta
$$

Write $\nu=\nu_{1}+\nu_{2}$, where $\operatorname{supp} \nu_{1} \subseteq\left\{\varsigma \in \mathrm{UHP}:\left|\varsigma-x_{0}\right| \leq 2 R\right\}$ and $\nu_{2}$ is supported in the complement. The $\nu_{2}$ role in (2.12) can be estimated directly, using (2.10). Because

$$
\int_{\left|\varsigma-x_{0}\right| \leq 2 R}|\nu(\varsigma)|^{2} \eta^{-1} d \xi d \eta \leq C R
$$

by assumption, the $\nu_{1}$ part of (2.12) is estimated using the following well-known result. 
LEMMA 2.13. The operator

$$
A f(\varsigma)=\int_{\mathrm{UHP}} \frac{\eta^{1 / 2} v^{1 / 2}}{|\varsigma-\bar{w}|^{3}} f(w) d u d v
$$

is bounded on $L^{2}$ (UHP, $\left.d x d y\right)$.

The $\nu_{1}$ part of (2.12) is at most $\int|A f|^{2} d \xi d \eta$, where $f(w)=\nu_{1}(w) v^{-1 / 2}$, and the lemma gives the estimate we wanted.

The lemma is proved using Schur's lemma. Observe that

$$
\int_{\mathrm{UHP}} \frac{\eta^{1 / 2} v^{1 / 2}}{|\zeta-\bar{w}|^{3}} d u d v \leq C .
$$

It is enough to check this for $\varsigma=i$, by homogeneity, and that is easy. One has the same inequality when integrating in $\zeta$. By Jensen's inequality, and then Fubini,

$$
\begin{aligned}
\int_{\mathrm{UHP}}|A f(\varsigma)|^{2} d \xi d \eta & \leq \int_{\mathrm{UHP}} C \int_{\mathrm{UHP}} \frac{\eta^{1 / 2} v^{1 / 2}}{|\zeta-\bar{w}|^{3}}|f(w)|^{2} d u d v d \xi d \eta \\
& \leq C \int_{\mathrm{UHP}}|f(w)|^{2} d u d v
\end{aligned}
$$

This finishes the proof that $\left|T\left(\nu_{z}\right)\right|^{2} y d x d y$ is a 2-GCM on the UHP when $\Gamma=\mathbf{R}$ and $\operatorname{supp} \nu \subseteq$ UHP. The above argument simplifies when estimating $T\left(\nu_{z}\right)$ on the LHP. In that case it is unnecessary to split $\partial F$ into $G_{1}$ and $G_{2}$ or to introduce the cutoff function $\varphi_{\zeta}(w)$, because the kernel no longer has a singularity. Instead, $T\left(\nu_{z}\right)$ is reduced directly by integration by parts to

$$
\int_{\mathbf{C}} \frac{1}{|\varsigma-w|^{3}}|\nu(w)| d u d v, \quad \varsigma \in \mathrm{LHP}, w \in \mathrm{UHP} .
$$

This is treated just like (2.10).

The case $\Gamma=\mathbf{R}$, supp $\nu \subseteq$ LHP is the same.

Similar arguments apply when $\Gamma$ is a general chord-arc curve. Many of the estimates can be reduced to the line using a bilipschitz map $\theta$ on $\mathbf{C}$ that takes $\mathbf{R}$ to $\Gamma$. Some minor modifications must be made, e.g., in $\varphi_{\varsigma}(w)=\varphi((w-\varsigma) / 10 \eta), \eta$ should be replaced by $\delta_{\Gamma}(\varsigma)$. In $(2.10), \bar{w}$ should be replaced by $w^{*}$, where $w \mapsto w^{*}$ is a bilipschitz reflection across $\Gamma$ that leaves every point of $\Gamma$ fixed. (This can be easily obtained using $\theta$ above.) The details are left to the reader.

This completes the proof of Lemma 2.3.

3. Theorem 0.1 in the general case using the Cauchy integral. Let us first review what the Cauchy integral is. Let $\Gamma$ be a rectifiable Jordan curve passing through $\infty$ with complementary regions $\Omega_{+}$and $\Omega_{-}$. Let $f$ be a function on $\Gamma$. We define its Cauchy integral by

$$
F(z)=\frac{1}{2 \pi i} \int_{\Gamma} \frac{f(w)}{w-z} d w, \quad z \notin \Gamma .
$$

If $f_{+}$and $f_{-}$denote the boundary values of $F_{ \pm}=\left.F\right|_{\Omega_{ \pm}}$, then the Plemelj formula states that

$$
f_{ \pm}(z)= \pm \frac{1}{2} f(z)+\frac{1}{2 \pi i} \mathrm{P} . \mathrm{V} . \int_{\Gamma} \frac{f(w)}{w-z} d w, \quad z \in \Gamma
$$

This singular integral is also called the Cauchy integral. 
In particular, the jump of $F$ across $\Gamma$, i.e., $f_{+}-f_{-}$, equals $f$. This property and the analyticity of $F$ off $\Gamma$ are simultaneously expressed in the equation $\bar{\partial} F=$ $f d z_{\Gamma}$, interpreted in the sense of distributions, where $d z_{\Gamma}$ denotes the measure on $\Gamma$ inducing the line integral $\int_{\Gamma} d z$. This $\bar{\partial}$ equation and a mild growth condition at $\infty$ characterize $F$.

Now suppose that $\Gamma_{0}, \mu$, and $\rho$ are as in Theorem 0.1. Assume also that $\mu$ is smooth and has compact support disjoint from $\Gamma_{0}$, so that $\rho$ is smooth. Let $\Gamma_{1}=\rho\left(\Gamma_{0}\right)$. We wish to compare the Cauchy integral on $\Gamma_{0}$ with the pull-back via $\rho$ of the Cauchy integral on $\Gamma_{1}$. Let $f$ be a function on $\Gamma_{0}$. The Cauchy integral $F_{1}$ of $f \circ \rho^{-1}$ on $\Gamma_{1}$ satisfies $\bar{\partial} F_{1}=0$ off $\Gamma_{1}$ and $F_{1}$ has jump $f \circ \rho^{-1}$ on $\Gamma_{1}$. Thus $G=F_{1} \circ \rho$ satisfies $(\bar{\partial}-\mu \partial) G=0$ off $\Gamma_{0}$ and its jump across $\Gamma_{0}$ is given by $f$.

Let $H=G-F$, where $F$ is the Cauchy integral of $f$ on $\Gamma_{0}$. Then $H$ has no jump across $\Gamma_{0}$, and $(\bar{\partial}-\mu \partial) H=\mu F^{\prime}$ off $\Gamma_{0}$. Because $H$ has no jump, this equation holds on all of $\mathbf{C}$, in the distributional sense; there is no boundary term for $H$, as there is for $\bar{\partial} F$. Thus $H=(\bar{\partial}-\mu \partial)^{-1}\left(\mu F^{\prime}\right)$. By the a priori assumptions on $\mu$, $\mu F^{\prime}$ is smooth and has compact support, and so we can always find $H$ such that $\nabla H \in L^{2}, H \in C^{\infty}, H$ is holomorphic in a neighborhood of $\infty$, and $H$ vanishes at $\infty$, just as in the remarks after Proposition 2.4.

The point is to get estimates. By Theorem 6.7 of [S3], if $f \in \operatorname{BMO}\left(\Gamma_{0}\right)$, then $\left.H\right|_{\Gamma_{0}}$ has BMO norm at most $C\|\mu\|_{M+N}\|f\|_{*}$ if $\|\mu\|_{M+N}$ is small enough. (Note that $\S 6$ of [S3] simplifies in our situation, because the a priori assumptions on $\mu$ get rid of distributional technical problems, such as defining $\mu F^{\prime}$ on C.)

It turns out to be more convenient for us to work with $L^{2}$ instead of BMO, because the latter ignores constants. (Any $L^{p}, 1<p<\infty$, would work as well.) As pointed out in $\S 11$ of [S3], $\S 6$ can be modified to work for $L^{p}$. In particular, if $f \in L^{2}\left(\Gamma_{0}\right)$ and $\|\mu\|_{M+N}$ is small enough, then $\left\|\left.H\right|_{\Gamma_{0}}\right\|_{2} \leq C\|\mu\|_{M+N}\|f\|_{2}$.

In order to use this to control $\rho$ we need to convert it into a statement about singular integrals. The boundary values of $F$ are given by (3.2), and those of $G$ by

$$
g_{ \pm}(z)= \pm \frac{1}{2} f(z)+\frac{1}{2 \pi i} \mathrm{P} . \mathrm{V} . \int_{\Gamma_{0}} \frac{f(w)}{\rho(w)-\rho(z)} d \rho(w), \quad z \in \Gamma_{0}
$$

Because $\mu=0$ on $\Gamma_{0}, \rho$ is holomorphic on $\Gamma_{0}$ and $d \rho(w)=\rho^{\prime}(w) d w$. The $L^{2}$ estimate on $\left.H\right|_{\Gamma_{0}}=\left.(G-F)\right|_{\Gamma_{0}}$ implies that

$$
K_{0} f(z)=\mathrm{P} . \mathrm{V} . \int_{\Gamma_{0}} \frac{f(w)}{w-z} d w \quad \text { and } \quad K f(z)=\mathrm{P} . \mathrm{V} . \int_{\Gamma_{0}} \frac{f(w) \rho^{\prime}(w) d w}{\rho(w)-\rho(z)}
$$

$z \in \Gamma_{0}$, satisfy $\left\|K-K_{0}\right\| \leq C\|\mu\|_{M+N}$ as operators on $L^{2}\left(\Gamma_{0}\right)$.

Because $K_{0}$ is bounded on $L^{2}(\Gamma)[\mathbf{D}, \mathbf{C D M}],\|K\| \leq C$ if $\|\mu\|_{M+N}$ is small enough. Let us use this to estimate $\left|\rho^{\prime}\right|$ on $\Gamma_{0}$ and the chord-arc constant of $\Gamma_{1}$. The idea is as follows. Fix an arc of $\Gamma_{0}$, and set $f=\overline{\rho^{\prime}}$ on that arc and zero elsewhere. Then evaluate $K f$ on an arc far enough away from the first one so that $\rho(w)-\rho(z)$ is roughly constant if $z$ and $w$ come from the two arcs, but not too far away, so that $\rho(w)-\rho(z)$ is not too small. This can be done because $\rho$ is quasiconformal. Using $\|K f\|_{2} \leq C\|f\|_{2}$ we will get estimates on $\rho^{\prime}$.

Let $I$ be any arc on $\Gamma_{0}$, with endpoints $a$ and $b$. For $z_{1}, z_{2} \in \Gamma_{0}$, let $A_{0}\left(z_{1}, z_{2}\right)$ denote the arc of $\Gamma_{0}$ that joins them, and similarly for $\Gamma_{1}$ and $A_{1}(\cdot, \cdot)$. Because 
$\|\mu\|_{M+N}$ is small, we can assume that $\|\mu\|_{\infty} \leq \frac{1}{2}$. Using the distortion theorems for q.c. maps (see [JKK]) we can find absolute constants $C_{1}, C_{2}, C_{3}$, and $C_{4}$, and points $z_{1}, z_{2} \in \Gamma_{0}$ so that if $D=\operatorname{diam} \rho(I)=\operatorname{diam}(A(\rho(a), \rho(b)))$, then

(3.3)(i) $D \leq C_{1}|\rho(a)-\rho(b)|$;

(ii) $|a-b| \geq\left|z_{1}-z_{2}\right| \geq C_{2}^{-1}|a-b|$;

(iii) $\operatorname{diarn}\left(A_{1}\left(\rho\left(z_{1}\right), \rho\left(z_{2}\right)\right)\right) \leq D$;

(iv) $1000 D \leq \operatorname{dist}\left(A_{1}\left(\rho\left(z_{1}\right), \rho\left(z_{2}\right)\right), A_{1}(\rho(a), \rho(b))\right) \leq C_{3} D$;

(v) $1000 \operatorname{diam}(I) \leq \operatorname{dist}\left(A_{0}\left(z_{1}, z_{2}\right), I\right) \leq C_{4} \operatorname{diam}(I)$.

Thus $I$ and $A_{0}\left(z_{1}, z_{2}\right)$ will be the two arcs discussed in the preceding paragraph.

From (3.3) it follows that there is a real number $\alpha$ such that if $w \in I$ and $z \in A_{0}\left(z_{1}, z_{2}\right)$, then

$$
\left|e^{i \alpha}-\frac{\rho(w)-\rho(z)}{|\rho(w)-\rho(z)|}\right| \leq \frac{1}{50} .
$$

Define $f \in L^{2}\left(\Gamma_{0}\right)$ by $f(w)=\overline{\rho^{\prime}(w) d w} /|d w|$ if $w \in I$ and $f(w)=0$ elsewhere. Here $|d w|$ denotes arclength measure, so that $d w=(d w /|d w|)|d w|$. Then

$$
\begin{aligned}
C \int_{I}\left|\rho^{\prime}(w)\right|^{2}|d w| & \geq C\|f\|_{2}^{2} \geq\|K f\|_{2}^{2} \\
& \geq \int_{A_{0}\left(z_{1}, z_{2}\right)}\left|\int_{I} \frac{\left|\rho^{\prime}(w)\right|^{2}|d w|}{\rho(w)-\rho(z)}\right|^{2}|d z| \\
& \geq C^{-1} \int_{A_{0}\left(z_{1}, z_{2}\right)}\left(\int_{I} \frac{\left|\rho^{\prime}(w)\right|^{2}|d w|}{|\rho(w)-\rho(z)|}\right)^{2}|d z| \\
& \geq C^{-1} \frac{|a-b|}{|\rho(a)-\rho(b)|^{2}}\left(\int_{I}\left|\rho^{\prime}(w)\right|^{2}|d w|\right)^{2} .
\end{aligned}
$$

For the last inequality we used (3.3), more than once, and the chord-arc condition on $\Gamma_{0}$. Thus

$$
\int_{I}\left|\rho^{\prime}(w)\right|^{2}|d w| \leq C \frac{|\rho(a)-\rho(b)|^{2}}{|a-b|}
$$

and so by Schwarz's inequality,

$$
\int_{I}\left|\rho^{\prime}(w)\right||d w| \leq C|\rho(a)-\rho(b)| .
$$

This implies that $\Gamma_{1}=\rho\left(\Gamma_{0}\right)$ is a chord-arc curve, with uniformly bounded chordarc constant (if $\|\mu\|_{M+N}$ is small).

To finish the proof of Theorem 0.1, we want to show that (1.2) holds with $v$ replaced by $\rho^{\prime}, \mathbf{R}$ replaced by $\Gamma_{0}$, and $\delta \leq C\|\mu\|_{M+N}$. We will do the same sort of thing as above, but using $\left\|K-K_{0}\right\| \leq C\|\mu\|_{M+N}$ instead of $\|K\| \leq C$. Let $a, b, I, z_{1}$, and $z_{2}$ be as above.

If we replace $\rho$ by $\alpha \rho+\beta, \alpha, \beta \in \mathbf{C}$, then nothing changes, neither the dilatation of $\rho$ nor the operator $K$. Thus we may assume that $\rho(a)=a$ and $\rho(b)=b$. Let us first get some control on $\rho(w)-w$ for nearby $w$.

We want to show that

$$
|\rho(w)-w| \leq C\|\mu\|_{\infty}|a-b|
$$


for $w \in A_{0}(a, b) \cup A_{0}\left(z_{1}, z_{2}\right)$. When $\|\mu\|_{\infty} \leq \frac{1}{2}$ the left side is at most $C|a-b|$, by the distortion theorem. When $\mu=0$, the left side is 0 . We now use a well-known Schwarz' lemma trick. If we replace $\mu,\|\mu\|_{\infty} \leq \frac{1}{2}$, by $z \mu,|z| \leq 1$, then $\rho$ depends holomorphically on $z$, and (3.5) follows from Schwarz' lemma. (We could also have estimated $|\rho(w)-w|$ in terms of $\|\mu\|_{M+N}$, which is good enough here, by computing $\left(K-K_{0}\right) f$ explicitly when $f$ is the characteristic function of an arc.)

We are now ready to verify (1.2) in our situation. Let $f$ be any function supported on $A_{0}(a, b)$ such that $|f| \leq 1$. Writing $\|\mu\|$ for $\|\mu\|_{M+N}$ and using (3.3), we have that

$$
\begin{aligned}
C\|\mu\| & |a-b|^{1 / 2} \geq C\|\mu\|\|f\|_{2} \geq\left\|\left(K-K_{0}\right) f\right\|_{2} \\
& \geq C^{-1}|a-b|^{-1 / 2} \int_{A_{0}\left(z_{1}, z_{2}\right)}\left|K f-K_{0} f\right||d z| \\
& \geq C^{-1} \int_{A_{0}\left(z_{1}, z_{2}\right)}|a-b|^{-1 / 2} \cdot\left|\int_{A_{0}(a, b)}\left(\frac{1}{w-z}-\frac{\rho^{\prime}(w)}{\rho(w)-\rho(z)}\right) f(w) d w\right||d z| .
\end{aligned}
$$

From (3.5), (3.4 $\left.\frac{1}{2}\right)$, and (3.3) we obtain

$$
\begin{aligned}
& \int_{A_{0}\left(z_{1}, z_{2}\right)}\left|\int_{A_{0}(a, b)}\left(\frac{\rho^{\prime}(w)}{\rho(w)-\rho(z)}-\frac{\rho^{\prime}(w)}{w-z}\right) f(w) d w\right||d z| \\
& \quad \leq \int_{A_{0}\left(z_{1}, z_{2}\right)} \int_{A_{0}(a, b)} \frac{|\rho(w)-w+\rho(z)-z|}{|w-z||\rho(w)-\rho(z)|}\left|\rho^{\prime}(w)\right||d w||d z| \\
& \quad \leq \int_{A_{0}\left(z_{1}, z_{2}\right)} \int_{A_{0}(a, b)} C\|\mu\|_{\infty}|a-b|^{-1}\left|\rho^{\prime}(w)\right||d w||d z| \\
& \quad \leq C\|\mu\|_{\infty}|\rho(a)-\rho(b)|=C\|\mu\|_{\infty}|a-b| .
\end{aligned}
$$

Plugging this into the preceding gives

$$
\int_{A_{0}\left(\boldsymbol{z}_{1}, z_{2}\right)}\left|\int_{A_{0}(a, b)}\left(\frac{\rho^{\prime}(w)-1}{w-z}\right) f(w) d w\right||d z| \leq C\|\mu\||a-b| .
$$

Taking

$$
f(w)=\frac{\rho^{\prime}(w)-1}{\left|\rho^{\prime}(w)-1\right|} \frac{d w}{|d w|}
$$

yields

$$
\int_{A_{0}\left(z_{1}, z_{2}\right)}\left|\int_{A_{0}(a, b)} \frac{\left|\rho^{\prime}(w)-1\right|}{w-z}\right| d w|||d z| \leq C\|\mu\||a-b| .
$$

As with (3.4), we have from (3.3) that for some $\beta \in \mathbf{R}$,

$$
\left|e^{i \beta}-\frac{w-z}{|w-z|}\right|<\frac{1}{50} \text { if } z \in A_{0}\left(z_{1}, z_{2}\right), w \in A_{0}\left(w_{1}, w_{2}\right)
$$

Hence

$$
\begin{aligned}
\int_{A_{0}(a, b)} \frac{\left|\rho^{\prime}(w)-1\right|}{|w-z|}|d w| & \leq C \operatorname{Re} \int_{A_{0}(a, b)} e^{i \beta} \frac{\left|\rho^{\prime}(w)-1\right|}{w-z}|d w| \\
& \leq C\left|\int_{A_{0}(a, b)} \frac{\left|\rho^{\prime}(w)-1\right|}{w-z}\right| d w||
\end{aligned}
$$


From this and (3.3) we get

$$
\begin{aligned}
\int_{A_{0}(a, b)}\left|\rho^{\prime}(w)-1\right||d w| & \leq C \int_{A_{\mathrm{C}}\left(z_{1}, z_{2}\right)} \int_{A_{0}(a, b)} \frac{\left|\rho^{\prime}(w)-1\right|}{|w-z|}|d w||d z| \\
& \leq C\|\mu\||a-b| .
\end{aligned}
$$

Because of the normalization $\rho(a)=a$ and $\rho(b)=b$, we can write this as

$$
\begin{aligned}
\int_{A_{0}(a, b)}\left|\frac{\rho(b)-\rho(a)}{b-a}-\rho^{\prime}(w)\right||d w| & \leq C\|\mu\||\rho(a)-\rho(b)| \\
& \leq C\|\mu\| \int_{A_{0}(a, b)}\left|\rho^{\prime}(w) \| d w\right|
\end{aligned}
$$

This is still valid if we remove the normalization $\rho(a)=a, \rho(b)=b$. Thus (1.2) holds, with $\delta=C\|\mu\|$. In view of Lemma 1.6, we have finished the proof of Theorem 0.1 .

4. Extensions of strongly q.s. embeddings and Theorem 0.2 . This section is broken into three subsections. In the first we use (a slight variant of) the Beurling-Ahlfors formula for strongly q.s. embeddings of $\mathbf{R}$ into $\mathbf{C}$ that are a small perturbation of the identity. In the second the general case is done using ideas of Tukia [Tu 2], and in the third we give a formula for small perturbations of the identity on a given chord-arc curve (instead of the line). This last is important for $\S 6$.

4i. Small perturbations of the identity on $\mathbf{R}$. Suppose $r: \mathbf{R} \rightarrow \mathbf{C}$ is given by $r(t)=r(0)+\int_{0}^{t} e^{a(u)} d u$, where $a \in \mathrm{BMO}$ is complex-valued, $\|a\|_{*}$ small. Set $a=\alpha+i \beta, h(t)=\int_{0}^{t} e^{\alpha(u)} d u$, and $b=\beta \circ h^{-1}$. Because $\|a\|_{*}$ is small, $h^{\prime}$ and $\left(h^{-1}\right)^{\prime}$ are $A_{\infty}$ weights, and $\|b\|_{*} \leq C\|\beta\|_{*} \leq C\|a\|_{*}$. Thus $z(t)=r(0)+\int_{0}^{t} e^{i b(u)} d u$ is the arc-length parameterization of a chord-arc curve with small constant, and $r=z \circ h$.

Let $\varphi$ and $\psi$ be $C^{\infty}$ functions supported on $[-1,1], \varphi$ even, $\psi$ odd, $\varphi(x) d x=1$, $\int \psi(x) x d x=1$. Set $f_{y}(x)=|y|^{-1} f\left(|y|^{-1} x\right)$, and define $\rho: \mathbf{C} \rightarrow \mathbf{C}$ by

$$
\begin{aligned}
& \rho(x, y)=\varphi_{y} * r(x)+i(\operatorname{sgn} y) \psi_{y} * r(x), \quad y \neq 0, \\
& \rho(x, 0)=r(x) .
\end{aligned}
$$

An important fact is that $\rho(z) \equiv z$ if $r(x) \equiv x$.

PROPOSITION 4.2. There is a $\gamma_{0}>0$ such that if $\|a\|_{*} \leq \gamma_{0}$, then $\rho$ is a quasiconformal map of $\mathbf{C}$ onto itself with the following properties. Its dilatation $\mu$ satisfies $\|\mu\|_{\infty} \leq C\|a\|_{*}$, and for $j \geq 0,\left|y^{j} \nabla^{j} \mu\right|^{2}|y|^{-1} d x d y$ is a Carleson measure with norm $\leq C_{j}\|a\|_{*}^{2}$, and $\left|y^{j} \nabla^{j} \mu(z)\right| \leq C_{j}\|a\|_{*}$. For $j \geq 2$, $\left|y^{j-1}\left(\nabla^{j} \rho\right)(\partial \rho)^{-1}\right|^{2}|y|^{-1} d x d y$ is a Carleson measure and $|y|^{j-1}\left|\nabla^{j} \rho \| \partial \rho\right|^{-1}$ is bounded, both with estimates in $\|a\|_{*}$.

In the case where $a$ is purely imaginary this follows from Proposition 2.5 in [S3]. Let us indicate how its proof can be extended to this more general situation. 
We shall repeatedly use certain well-known BMO calculations. For example, if $\|f\|_{*}$ is small, then $\left|\varphi_{y} * e^{f}\right|$ is comparable to $\left|\exp \left(\varphi_{y} * f\right)\right|$. Indeed, since $\int \varphi=1$,

(b) $\left|\left(\varphi_{y} * e^{f}\right)(x)-e^{\varphi_{y} * f(x)}\right| \leq C\left|e^{\varphi_{y} * f(x)}\right| \frac{1}{2 y} \int_{x-y}^{x+y}\left|e^{f(u)-f * \varphi_{y}(x)}-1\right| d u$,

$$
\left|\varphi_{y} * f(x)-\frac{1}{2 y} \int_{x-y}^{x+y} f(u) d u\right| \leq C\|f\|_{*},
$$

one gets from John-Nirenberg and $\left|e^{x}-1\right| \leq|x|\left|e^{x}\right|$ that

$$
(2 y)^{-1} \int_{x-y}^{x+y}\left|e^{f(u)-f * \varphi_{y}(x)}-1\right| d u \leq C\|f\|_{*}
$$

if $\|f\|_{*}$ is small enough. In particular, $\left|\exp \left(\varphi_{y} * f\right)\right|$ and $\left|\varphi_{y} * e^{f}\right|$ are comparable if $\|f\|_{*}$ is small. Also,

$$
\left|\exp \left(\varphi_{y} * f(x)\right)\right| \leq C\left|\exp \left(\varphi_{y} * f\left(x^{\prime}\right)\right)\right| \quad \text { if }\left|x-x^{\prime}\right| \leq y
$$

Consider $\mu$ on the UHP. (The LHP is treated similarly.) As in [S3], $\bar{\partial} \rho(x, y)=$ $\nu_{y} * r^{\prime}$ and $\partial \rho=\beta_{y} * r^{\prime}$ where $\int \nu=0$ and $\int \beta=1$, and $\nu$ and $\beta$ are $C^{\infty}$ and supported on $[-1,1]$. (This reflects the fact that $\rho(z) \equiv z$ if $r(x) \equiv x$.) Thus

$$
\begin{aligned}
|\mu(x, y)| & =\left|\frac{\bar{\partial} \rho}{\partial \rho}\right| \leq C\left|\left(\nu_{y} * e^{a}\right)(x)\right|\left|\exp \left(-\beta_{y} * a(x)\right)\right| \\
& =C\left|\left(\nu_{y} * e^{a-\beta_{y} * a(x)}\right)(x)\right| \\
& =C\left|\nu_{y} *\left(e^{a-\beta_{y} * a(x)}-1\right)(x)\right| \leq C\|a\|_{*}
\end{aligned}
$$

if $\|a\|_{*}$ is small enough, by (4.4).

Let us show that $|\mu|^{2} y^{-1} d x d y$ is a Carleson measure. For each interval $I$ we must estimate

$$
\int_{I} \int_{0}^{|I|}|\mu(x, y)|^{2} y^{-1} d x d y
$$

We may assume that $\int_{5 I} a=0$, because adding a constant to $a$ does not change $\mu$. Since $\left|\beta_{y} * e^{a}\right|^{-1} \approx C\left|e^{-\beta_{y} * a}\right| \approx C\left|\beta_{y} * e^{-a}\right|$,

$$
\begin{aligned}
\int_{I} \int_{0}^{|I|}|\mu(x, y)|^{2} y^{-1} d x d y \\
\leq C \int_{I} \int_{0}^{|I|}\left|\left(\nu_{y} * e^{a}\right)(x)\right|^{2}\left|\beta_{y} * e^{-a}(x)\right|^{2} y^{-1} d x d y \\
\leq C\left(\int_{I 0<y<|I|}\left|\beta_{y} * e^{-a}(x)\right|^{4} d x\right)^{1 / 2} \\
\\
\cdot\left(\int_{I}\left(\int_{0}^{|I|}\left|\nu_{y} * e^{a}(x)\right|^{2} \frac{d y}{y}\right)^{2} d x\right)^{1 / 2} .
\end{aligned}
$$


Because $\operatorname{supp} \beta, \operatorname{supp} \nu \subseteq[-1,1]$, we can replace $a$ by $a \chi_{5 I}$, and because $\int \nu=0$, we can replace $\nu_{y} * e^{a}$ by $\nu_{y} *\left(\left(e^{a}-1\right) \chi_{5 I}\right)$. Since the maximal and LittlewoodPaley functions $\sup _{y>0}\left|\beta_{y} * f(x)\right|$ and $\left(\int_{0}^{\infty}\left|\nu_{y} * f(x)\right|^{2} d y / y\right)^{1 / 2}$ are bounded on $L^{p}$, $1<p<\infty$ (see [Je or $\mathbf{S t}]$ ), the above is at most

$$
\left(\int_{5 I}\left|e^{-a(x)}\right|^{4} d x\right)^{1 / 2}\left(\int_{5 I}\left|e^{a}-1\right|^{4} d x\right)^{1 / 2} .
$$

Using John-Nirenberg, the smallness of $\|a\|_{*}$, and $\int_{5 I} a=0$, we obtain

$$
\leq C|I|^{1 / 2}\|a\|_{*}^{2}|I|^{1 / 2} \leq C|I|\|a\|_{*}^{2} .
$$

Thus the Carleson measure norm of $|\mu|^{2} y d x d y$ is at most $C\|a\|_{*}^{2}$.

The estimates for the higher derivatives of $\mu$ and $\rho$ are similar, and we omit the details. (The reader may find $\S \S 1$ and 2 in [S3] helpful for this.) We are left with showing that $\rho$ is a homeomorphism on $\mathbf{C}$. One could mimic the proof of bilipschitzness in $[\mathbf{S 3}]$ when $\operatorname{Re}(a) \equiv 0$, but for simplicity we give a less direct proof.

Suppose first that $a$ is smooth and has compact support. Let us check that $\rho$ must then be $C^{\mathbf{1}}$. It is always smooth off $\mathbf{R}$. Because $\bar{\partial} \rho(x, y)=\nu_{y} * r^{\prime}(x)$ and $\partial \rho(x, y)=\beta_{y} * r^{\prime}(x), \int \nu=0, \int \beta=1$, and $r^{\prime}$ is continuous, it follows that $\bar{\partial} \rho$ and $\partial \rho$ are continuous on $\mathbf{R}$, where they take the values 0 and $r^{\prime}(x)$, respectively. Since our earlier estimates give that $\mu$ is small and $\partial \rho$ is never $0, \rho$ is locally a homeomorphism on $C$, and is also an open mapping, by the inverse function theorem. Since $a$ has compact support, $r(x)=x+o(x)$ at $\infty$, and $\rho(z)=z+o(z)$ at $\infty$. Hence $\rho(z) \rightarrow \infty$ as $z \rightarrow \infty$, and standard topological arguments (i.e., the monodromy theorem) imply that $\rho$ is a homeomorphism of $\mathbf{C}$ onto itself, and is hence quasiconformal.

Consider now the general case. Given $a \in \mathrm{BMO},\|a\|_{*}$ small enough, we can find $a_{j}$, smooth and compactly supported, such that $\left\|a_{j}\right\|_{*} \leq C\|a\|_{*}, a_{j} \rightarrow a$ a.e. and locally in $L^{1}, e^{a_{j}} \rightarrow e^{a_{j}}$ locally in $L^{1}$, and $\int_{0}^{1} a_{j}=\int_{0}^{1} a$ for all $j$. The corresponding $\rho_{j}$ are quasiconformal with small dilatation, by the above arguments. If we require that $r_{j}(0)=r(0)$ for all $j$, then $r_{j} \rightarrow r$ uniformly on compact sets, and $\rho_{j} \rightarrow \rho$ does too. Because of our normalizations we can conclude that $\rho$ is quasiconformal. This proves the proposition.

4ii. The general case. Suppose $r: \mathbf{R} \rightarrow \mathbf{C}$ is strongly q.s., so that $\Gamma=r(\mathbf{R})$ is a chord-arc curve and $r(x)=z(h(x))$, where $z(\cdot)$ is an arclength parameterization of $\Gamma$ and $h^{\prime}(x)=\left|r^{\prime}(x)\right| \in A_{\infty}$. We want to find a well-behaved q.c. extension $\rho$ of $r$, so that $\rho$ in particular satisfies the conclusions of Theorem 0.2 .

Let $\Omega_{+}$and $\Omega_{-}$denote the complementary regions of $\Gamma$, and let $\Phi_{+}$and $\Phi_{-}$be conformal maps of the UHP and LHP onto $\Omega_{+}$and $\Omega_{-}$that take $\infty$ to itself. Define increasing homeomorphisms $h_{+}$and $h_{-}$on $\mathbf{R}$ by $\Phi_{ \pm}(x)=z\left(h_{ \pm}(x)\right)$. Lavrentiev's theorem states that $h_{ \pm}^{\prime} \in A_{\infty}$ (see $[\mathbf{J K}]$ ).

Let $k_{ \pm}=h_{ \pm}^{-1} \circ h$, so that $r=\Phi_{ \pm} \circ k_{ \pm}$. To get the desired extension $\rho$ of $r$ we follow Tukia [Tu 2] and first find good extensions of $k_{+}$and $k_{-}$and then compose with $\Phi_{+}$and $\Phi_{-}$. A smoothed up version of the Beurling-Ahlfors exension could be used, but for technical reasons it is convenient to break up $k_{ \pm}$into small pieces beforehand. 
Suppose $k(x)$ is an increasing locally absolutely continuous homomorphism on $\mathbf{R}$ such that $k^{\prime} \in A_{\infty}$. Define $k_{t}$ by $k_{t}(x)=k(0)+\int_{0}^{x} k^{\prime}(u)^{t} d u, 0 \leq t \leq 1$. Then $k_{t}^{\prime} \in A_{\infty}$, with constants independent of $t$, so that $f \mapsto f \circ k_{t}^{-1}$ defines a uniformly bounded family of operators on $\mathrm{BMO}(\mathbf{R})$. For $s<t$,

$$
\begin{aligned}
\left(k_{t} \circ\left(k_{s}^{-1}\right)\right)^{\prime}(x) & =k^{\prime}\left(k_{s}^{-1}(x)\right)^{t}\left[\left(k_{s}^{-1}\right)^{\prime}(x)\right] \\
& =k^{\prime}\left(k_{s}^{-1}(x)\right)^{t-s} .
\end{aligned}
$$

Thus

$$
\left\|\log \left(k_{t} \circ k_{s}^{-1}\right)^{\prime}\right\|_{*}=(t-s)\left\|\log k^{\prime} \circ k_{s}^{-1}\right\|_{*} \leq C(t-s)\left\|\log k^{\prime}\right\|_{*} .
$$

By taking $t-s$ sufficiently small we make $\left\|\log \left(k_{t} \circ k_{s}^{-1}\right)^{\prime}\right\|_{*}$ as small as we like. Because $k_{0}(x) \equiv x$ and $k_{1}=k$, we can write $k=k_{N} \circ k_{N-1} \circ \cdots \circ k_{2} \circ k_{1}$ with $\left\|\log k_{j}^{\prime}\right\|_{*}$ as small as we like for each $j$. ( $N$ will depend on how small we want it.)

Apply this to $k_{+}$and $k_{-}$to get $k_{+, j}$ and $k_{-, j}$. Proposition 4.2 gives extensions $\rho_{+, j}$ and $\rho_{-, j}$ of these. Define $\rho_{+}=\rho_{+, N} \circ \rho_{+, N-1} \circ \cdots \circ \rho_{+, 1}$, and similarly for $\rho_{-}$. Thus $\rho_{+}$and $\rho_{-}$are q.c. maps that extend $k_{+}$and $k_{-}$, and hence preserve the UHP and LHP. Define $\rho$ by $\rho=\Phi_{+} \circ \rho_{+}$on the UHP and $\rho=\Phi_{-} \circ \rho_{-}$on the LHP. Then $\rho$ is a q.c. map on $\mathbf{C}$ that extends $r$. We want to estimate its derivatives and dilatation, in particular their Carleson measure estimates. By symmetry it is enough to do this on the UHP. We first need to know when a q.c. change of variables is nice to Carleson measures.

Let $\theta$ be a q.c. map of the UHP onto itself. Consider the following properties:

(4.6)(a) $C^{-1} \leq y|\partial \theta| / \operatorname{Im} \theta \leq C, \theta=\theta(x, y)$;

(b) $\theta(x)=\theta(0)+\int_{0}^{x} w(t) d t, w \in A_{\infty}$.

The first one says that $\theta$ preserves the hyperbolic metric on the UHP to within constants.

If $\rho$ is defined by (4.1) with $r^{\prime}=e^{a},\|a\|_{*}$ small, $a$ real valued, then $\theta=\rho$ satisfies (a) and (b) above. Let us check (a); (b) is by definition.

As in the proof of Proposition 4.2, $|\partial \rho| \approx\left|\exp \left(\beta_{y} * a(x)\right)\right|$, where $\int \beta=1$. By definition, $\operatorname{Im} \rho(x, y)=\psi_{y} * r(x)$. The definition of $\psi$ implies that $\psi=\gamma^{\prime}$, where $\gamma$ is smooth, supp $\gamma \subseteq[-1,1]$, and $\int \gamma=1$. This implies that $\psi_{y}=|y|(d / d x) \gamma_{y}$, so that $\psi_{y} * r(x)=|y| \gamma_{y} * r^{\prime}(x)$. Since $\int \gamma=1,\left|\gamma_{y} * r^{\prime}(x)\right| \approx\left|\exp \left(\gamma_{y} * a(x)\right)\right|$, and this is comparable to $\left|\exp \left(\beta_{y} * a(x)\right)\right|$, because $\left\|\beta_{y} * a-\gamma_{y} * a\right\|_{L^{\infty}} \leq C\|a\|_{*}$, by (4.3)(c). Thus $\rho$ satisfies (4.6)(a).

If $\theta$ is a q.c. map of the UHP onto itself, the following is a consequence of (4.6)(a) and (b) (and is in fact equivalent to (4.6)(a) and (b)):

There is a $w \in A_{\infty}$ and a $C>0$ such that

$$
C^{-1}|\partial \theta(x, y)| \leq \frac{1}{y} \int_{x-y}^{x+y} w(t) d t \leq C|\partial \theta(x, y)| \quad \text { for all } x, y \in \mathbf{R} .
$$

Indeed, the distortion theorem for q.c. maps implies that

$$
\operatorname{Im} \theta(x+i y) \approx \theta(x+y)-\theta(x-y)=\int_{x-y}^{x+y} \theta^{\prime}(u) d u .
$$

Observe that if $\theta, \tilde{\theta}$ are two q.c. maps on the UHP that satisfy (4.6)(a) and (b), then so do $\theta^{-1}$ and $\theta \circ \tilde{\theta}$, i.e., they form a group. 
LEMMA 4.8. Let $\theta$ be a q.c. map of the UHP onto itself that satisfies (4.6)(a) and (b). Suppose that $\alpha(z)$ is locally integrable on the UHP and that $|\alpha(z)| d x d y$ is a Carleson measure. Then $|\alpha(\theta(z)) \| \partial \theta(z)| d x d y$ is also a Carleson measure, with norm dominated by the norm of $\alpha$.

This corresponds to the fact that if $h^{\prime} \in A_{\infty}$, then $f \rightarrow f \circ h$ preserves BMO.

Let us prove the lemma. Set $\beta(z)=\alpha(\theta(z))(\partial \theta(z))$ and let $x_{0} \in \mathbf{R}$ and $R>0$ be given. We want to show that

$$
\iint_{\substack{z \in \mathrm{UHP}^{\prime} \\\left|z-x_{0}\right|<R}}|\beta(z)| d x d y \leq C R .
$$

By the distortion theorems, if $\tilde{R}=\theta\left(x_{0}+R\right)-\theta\left(x_{0}-R\right)$, then

$$
\theta\left(\left\{z \in \mathrm{UHP}:\left|z-x_{0}\right| \leq R\right\}\right) \subseteq\left\{w \in \mathrm{UHP}:\left|w-\theta\left(x_{0}\right)\right| \leq C \tilde{R}\right\}
$$

for some $C>0$. Thus it is enough to show that

$$
\iint_{\substack{w \in \operatorname{UHP} \\-\theta\left(x_{0}\right) \mid \leq C \tilde{R}}}\left|\beta\left(\theta^{-1}(w)\right)\right| J_{\theta^{-1}}(w) d u d v \leq C R,
$$

where $J_{\theta^{-1}}$ denotes the Jacobian of $\theta^{-1}$. Because $\theta$ and $\theta^{-1}$ are q.c., $\left|J_{\theta^{-1}}\right| \approx$ $\left|\partial \theta^{-1}\right|^{2}$ and $\left|\partial \theta^{-1}\right| \approx\left|\partial \theta \circ \theta^{-1}\right|^{-1}$, and so the integral above is dominated by

$$
\iint_{\substack{w \in \mathrm{UHP} \\\left|w-\theta\left(x_{0}\right)\right| \leq C \tilde{R}}}\left|\partial\left(\theta^{-1}(w)\right) \| \alpha(w)\right| d u d v .
$$

Because $\theta^{-1}$ satisfies (4.6) and (4.7), there is an $A_{\infty}$ weight $w(x)$ so that

$$
\left|\partial\left(\theta^{-1}(z)\right)\right| \approx y^{-1} \int_{x-y}^{x+y} w(t) .
$$

If

$$
w^{*}(t)=\sup \left\{y^{-1} \int_{x-y}^{x+y} w(t) d t:|x-t| \leq y \leq C \tilde{R}\right\}
$$

then

$$
\begin{aligned}
\tilde{R}^{-1} \int_{\theta\left(x_{0}\right)-C \tilde{R}}^{\theta\left(x_{0}\right)+C \tilde{R}} w^{*}(t) d t & \leq C\left(\tilde{R}^{-1} \int_{\theta\left(x_{0}\right)-C \tilde{R}}^{\theta\left(x_{0}\right)+C \tilde{R}} w(t)^{1+\delta} d t\right)^{1 /(1+\delta)} \\
& \leq C\left(\tilde{R}^{-1} \int_{\theta\left(x_{0}\right)-\tilde{R}}^{\theta\left(x_{0}\right)+\tilde{R}} w(t) d t\right) \\
& \leq C \tilde{R}^{-1}\left(\theta^{-1}\left(\theta\left(x_{0}\right)+\tilde{R}\right)-\theta^{-1}\left(\theta\left(x_{0}\right)-\tilde{R}\right)\right) \\
& \leq C(\tilde{R})^{-1} R
\end{aligned}
$$

On the second inequality we have used the reverse Hölder inequality for $w(t)$ and also the fact that $w(t) d t$ is a doubling measure. In the last inequality we used the distortion theorem. 
This maximal function estimate for $\partial\left(\theta^{-1}\right)$ and the Carleson condition on $\alpha$ implies that (4.9) is $\leq C R$. This proves the lemma.

Notice that $|f(\theta(z))| y^{-1} d x d y$ is a Carleson measure if $|f(z)| y^{-1} d x d y$ is and if $\theta$ satisfies (4.6), by the lemma.

Theorem 0.2 is a consequence of the following.

PROPOSITION 4.10. Let $\rho$ be as above, and let $\mu$ be its dilatation. Then, for $j \geq$ $0,\left|y^{j} \nabla^{j} \mu\right|^{2}|y|^{-1} d x d y$ is a Carleson measure. Also, for $j \geq 2,|y|^{j-1}\left|\nabla^{j} \rho\right||\partial \rho|^{-1}$ is bounded, and $\left|y^{j-1}\left(\nabla^{j} \rho\right)(\partial \rho)^{-1}\right|^{2}|y|^{-1} d x d y$ is a Carleson measure.

It is enough to prove this on the UHP. From Proposition 4.2 we know that each $\rho_{+, j}$ satisfies the conclusions of Proposition 4.10, and also (4.6). Straightforward computation and Lemma 4.8 imply that $\rho_{+}=\rho_{+, N} \circ \cdots \circ \rho_{+, 1}$ has the same properties. (This should be checked one composition at a time.) As for $\rho=\Phi_{+} \circ \rho_{+}$, first observe that it has the same dilatation as $\rho_{+}$, because $\Phi_{+}$is conformal. The estimates on $\nabla^{j} \rho$ are obtained as above, using also the fact that $\log \Phi_{+}^{\prime} \in \mathrm{BMOA} \subseteq$ Bloch.

4iii. Small perturbations of the identity on a chord-arc curve. Let $\Gamma$ be a fixed chord-arc curve and let $z(\cdot)$ be a fixed arc-length parameterization of $\Gamma$. Let $a$ be a complex-valued $\mathrm{BMO}(\Gamma)$ function such that $\|a\|_{*}$ is small, and suppose that $r: \Gamma \rightarrow C$ satisfies $r(z)=r\left(z_{0}\right)+\int_{z_{0}}^{z} e^{a(w)} d w$ for $z, z_{0} \in \Gamma$, where the integral is taken along $\Gamma$. Thus if $a \equiv 0$, then $r$ is a translation. We want to find a q.c. extension $\rho$ of $r$ whose dilatation satisfies estimates analogous to those in Proposition 4.2. We shall give a formula similar to 4.1. First we need the following.

LEMMA 4.11. There is a bilipschitz mapping $\tau$ of $\mathbf{C}$ onto $\mathbf{C}$ such that $\tau(x)=$ $z(x), x \in \mathbf{R}$, and $\left|y^{j} \nabla^{j} \nu\right|^{2}|y|^{-1} d x d y$ is a Carleson measure for $j \geq 0$, where $\nu$ is the dilatation of $\tau$. Also, $\left|\nabla^{j} \tau\right| \leq C_{j}|y|^{j-1}, j \geq 1$.

To prove this we apply the argument in the preceding subsection to $r(t) \equiv z(t)$. Thus if $h, h_{+}, h_{-}, k_{+}, k_{-}, \Phi_{+}$, and $\Phi_{-}$are as before, then $h(x) \equiv x, k_{ \pm}=\left(h_{ \pm}\right)^{-1}$. We also define $\rho_{+}$and $\rho_{-}$as before and we take $\tau$ to be what we called $\rho$ before, i.e., $\tau=\Phi_{ \pm} \circ \rho_{ \pm}$on the UHP and LHP. All the desired properties of $\tau$ follow from Proposition 4.10, except the bilipschitzness.

It is enough to show that $C^{-1} \leq|\partial \tau| \leq C$. By symmetry, it suffices to do this on the UHP. Because $\partial \tau=\left(\Phi_{+}^{\prime} \circ \rho_{+}\right) \partial \rho_{+}$, it is enough to show that $\mid \Phi_{+}^{\prime}(z)\left(\partial \rho_{+}\right) \circ$ $\rho_{+}^{-1}(z)|=| \Phi_{+}^{\prime}(z)\left(\partial\left(\rho^{-1}\right)(z)\right)^{-1} \mid$ is bounded above and below. Since $\rho_{+}^{-1}$ satisfies (4.6)(a), the distortion theorems give

$$
\begin{aligned}
\left|\partial\left(\rho_{+}^{-1}\right)(z)\right| & \approx y^{-1} \operatorname{Im} \rho_{+}^{-1}(z) \approx y^{-1}\left(\rho_{+}^{-1}(x+y)-\rho_{+}^{-1}(x-y)\right) \\
& =y^{-1} \int_{x-y}^{x+y} h_{+}^{\prime}(t) d t
\end{aligned}
$$

the last equality from definition chasing.

Since $h_{+}^{\prime} \in A_{\infty}, h_{+}^{\prime} \in A_{p}$ for some $p<\infty$. This and Jensen's inequality give

$$
\frac{1}{2 y} \int_{x-y}^{x+y} h_{+}^{\prime}(t) d t \approx \exp \left(\frac{1}{2 y} \int_{x-y}^{x+y} \log h_{+}^{\prime}(t) d t\right) \text {. }
$$

From

$$
\left|\frac{1}{2 y} \int_{x-y}^{x+y} \log h_{+}^{\prime}(t) d t-\frac{1}{\pi} \int_{\mathbf{R}} \frac{y}{(x-t)^{2}+y^{2}} \log h_{+}^{\prime}(t) d t\right| \leq C\left\|\log h_{+}^{\prime}\right\|_{*}
$$


and the fact that $\log \left|\Phi_{+}^{\prime}(z)\right|$ is the Poisson integral of $\log \left|\Phi^{\prime}(x)\right|=\log h_{+}^{\prime}(x)$, we get that $\left|\Phi^{\prime}(z)\right| \approx\left|\partial\left(\rho_{+}^{-1}(z)\right)\right|$, as desired. Thus $\tau$ is bilipschitz. This proves the lemma.

Let $\Gamma, r$, and $a$ be as before the lemma. For the rest of this section we let $\tilde{z}(t)$ denote an arclength parameterization of $\Gamma$, so as not to confuse it with the complex variable $z=x+i y$. We want to find a formula for a good extension of $r$. This is trickier than (4.1). Set $b(t)=a(\tilde{z}(t))$ and $s(t)=r(\tilde{z}(t))$, so that $\|b\|_{*}$ is small and $s(t)=s(0)+\int_{0}^{t} e^{b(u)} \tilde{z}^{\prime}(u) d u$. Let $\varphi(x)$ be a smooth even function on $\mathbf{R}$ such that $\int \varphi=1$ and $\operatorname{supp} \varphi \subseteq[-1,1]$, and put $\varphi_{y}(x)=|y|^{-1} \varphi\left(|y|^{-1} x\right)$. We also want that $\left|\left(\varphi_{y} * \tilde{z}^{\prime}\right)(x)\right| \geq \varepsilon_{0}$ for some $\varepsilon_{0}>0$. This is achieved by requiring $\varphi-\chi_{[-1 / 2,1 / 2]}$ to have very small $L^{1}$ norm, since $\left|\tilde{z}^{\prime}(x)\right| \equiv 1$ and $\left|\int_{x}^{y} \tilde{z}^{\prime}(t) d t\right| \geq(1+k)^{-1}|x-y|$, by the chord-arc condition on $\Gamma$.

Define $P_{y} f=\varphi_{y} * f$ and $R_{y} f=\left(P_{y}\left(\tilde{z}^{\prime}\right)\right)^{-1} P_{y}\left(\tilde{z}^{\prime} f\right)$. Thus $R_{y}(1)=1$, so that $R_{y} f$, like $P_{y} f$, is an average of $f$ at the scale of $y$. Unlike $P_{y}$, it is "well adapted" to $\tilde{z}^{\prime}$. Such operators show up in [DJS] in a more complicated way. Here we shall need only easy properties of $R_{y}$.

Using $\tau(z)$ from Lemma 4.11, define

$$
\begin{aligned}
& \sigma(x, y)=\varphi_{y} * s(x)+R_{y}\left(e^{b}\right)(x)\left(\tau(x+i y)-\varphi_{y} * \tau(x)\right), \quad y \neq 0, \\
& \sigma(x, 0)=s(x) .
\end{aligned}
$$

Here $\varphi_{y} * \tau=\varphi_{y} *\left(\left.\tau\right|_{\mathbf{R}}\right)=\varphi_{y} * \tilde{z}$. Let $\rho=\sigma \circ \tau^{-1}$.

PROPOSITION 4.13. $\rho$ is a quasiconformal map of $\mathbf{C}$ onto itself. If $\mu$ denotes its dilatation, then for $j \geq 0,\left|\delta_{\Gamma}(z)^{j} \nabla^{j} \mu(z)\right|^{2} \delta_{\Gamma}(z) d x d y$ is a Carleson measure relative to $\Gamma$ with norm $\leq C_{j}\|a\|_{*}^{2}$, and $\left|\delta_{\Gamma}(z)^{j} \nabla^{j} \mu(z)\right| \leq C_{j}\|a\|_{*}$.

Let us motivate (4.12). Compare it to (4.1). The first terms are the same. By the definition of $\psi$ in (4.1), $\psi=\gamma^{\prime}$ where $\int \gamma=1$, so that the second term in (4.1) can be rewritten as $i(\operatorname{sgn} y) \psi_{y} * r=i y \gamma_{y} * r^{\prime}$. For the corresponding term in (4.12), we have replaced $i y$ by $\tau(x+i y)-\varphi_{y} * \tau(x)$. Notice that the two are equal if $\Gamma=\mathbf{R}$ and $\tau(z)=z$. In general, $\tau(x+i y)-\varphi_{y} * \tau(x)$ is roughly perpendicular to $\Gamma$ at $\tilde{z}(x)$ and is smooth at the scale of $y$. We use $R_{y}\left(e^{b}\right)$ instead of $\gamma_{y} * e^{b}$ in (4.12) because it gives better estimates. For example, it is responsible for $|\mu|^{2} \delta_{\Gamma}(z)^{-1} d x d y$ being a Carleson measure.

The geometrical motivation for (4.12) is the same as for the Beurling-Ahlfors formula. One has to compensate for the fact that $\Gamma$ is not a line, for which the bilipschitz map $\tau$ is helpful. The point is that on each arc of $\Gamma, r$ is roughly a combination of a translation, dilatation, and rotation. A good extension of $r$ should have the same property on any disk centered on $\Gamma$, where the choice of translation, dilatation, and rotation is forced by $r$. Both (4.1) and (4.12) have this property. (The reader should draw some pictures.)

Let us prove Proposition 4.13. First notice that $R_{y}$ has many properties in common with $P_{y}$. For example, (4.3) and (4.4) hold with $\varphi_{y} * f$ replaced by $R_{y} f$, and if $\|f\|_{*}$ is small enough, then $\left|R_{y}\left(e^{f}\right)\right|$ is comparable to $\left|\exp \left(R_{y} f\right)\right|$. (In fact, these properties hold for any decent approximation to the identity.) Also, $\left|y^{j} \nabla^{j}\left(R_{y}\left(e^{f}\right)(x)\right)\right| \leq C_{j}\|f\|_{*}\left|R_{y}\left(e^{f}\right)(x)\right|$ for $j \geq 1$. This last is because $y^{j} \nabla^{j} R_{y}(1)$ $\equiv 0$, so that (4.4) can be used. 
We also have the square function estimate

$$
\iint_{\mathbf{C}}\left|y^{j} \nabla^{j}\left(R_{y} f\right)(x)\right|^{2}|y|^{-1} d x d y \leq C_{j}\|f\|_{L^{2}(\mathbf{R})}^{2}, \quad j \geq 1 .
$$

We will do this for $j=1$ only; $j \geq 2$ is similar. Thus

$$
y \nabla R_{y} f=-y\left[\nabla\left(P_{y} \tilde{z}^{\prime}\right)\right]\left(P_{y} \tilde{z}^{\prime}\right)^{-2} P_{y}\left(\tilde{z}^{\prime} f\right)+P_{y}\left(\tilde{z}^{\prime}\right)^{-1} y \nabla P_{y}\left(\tilde{z}^{\prime} f\right) .
$$

The second term is controlled using the fact that $Q_{y} g=y \nabla P_{y} g$ satisfies

$$
\iint_{\mathbf{C}}\left|Q_{y} g\right|^{2}|y|^{-1} d x d y \leq C\|g\|_{2}^{2} .
$$

This can be derived using Plancherel; see [Je, Chapter 6], for example. The second term is controlled by

$$
\iint_{\mathbf{C}}\left|Q_{y}\left(z^{\prime}\right)\right|^{2}\left|P_{y}\left(z^{\prime} f\right)\right|^{2}|y|^{-1} d x d y
$$

which is at most $\|f\|_{2}^{2}$, since $\left|Q_{y}\left(z^{\prime}\right)\right|^{2}|y|^{-1} d x d y$ is a Carleson measure.

We need to estimate the derivatives of $\sigma$.

$$
\begin{aligned}
\bar{\partial} \sigma(z)= & \bar{\partial}\left(\varphi_{y} * s(x)\right)+\bar{\partial}\left(R_{y}\left(e^{b}\right)(x)\right)\left(\tau(z)-\varphi_{y} * \tau(x)\right) \\
& +R_{y}\left(e^{b}\right)(x) \bar{\partial} \tau(z)-R_{y}\left(e^{b}\right)(x) \bar{\partial}\left(\varphi_{y} * \tau(x)\right) .
\end{aligned}
$$

The third term is the main term. The second is at most $C|y|\left|\bar{\partial}\left(R_{y}\left(e^{b}\right)(x)\right)\right|$, since $\tau$ is bilipschitz. By our preceding remarks, this is $\leq C\|b\|_{*}\left|R_{y}\left(e^{b}\right)(x)\right|$.

Also,

$$
\left|R_{y}\left(e^{b}\right)(x)^{-1} y \bar{\partial}\left(R_{y}\left(e^{b}\right)(x)\right)\right|^{2}|y|^{-1} d x d y
$$

is a Carleson measure relative to $\mathbf{R}$, with norm $\leq C\|b\|_{*}^{2}$. Let $I$ be any interval, and let us see how the integral of (4.17) over $\hat{I}=\{z \in \mathrm{C}: x \in I,|y| \leq|I|\}$ is estimated. We may suppose that $\int_{5 I} b=0$, and we can replace $\bar{\partial}\left(R_{y}\left(e^{b}\right)(x)\right)$ by $\bar{\partial}\left(R_{y}\left(\left(e^{b}-1\right)(x)\right)\right.$. Expand $\bar{\partial}\left(R_{y}\left(e^{b}-1\right)(x)\right)$ as in (4.15). We can ignore the factors of $P_{y}\left(\tilde{z}^{\prime}\right)^{-1}$, since they are bounded. The contribution of the first term is

$$
\begin{aligned}
\iint_{\hat{I}}\left|R_{y}\left(e^{b}\right)^{-1} Q_{y}\left(\tilde{z}^{\prime}\right) P_{y}\left(\tilde{z}^{\prime}\left(e^{b}-1\right)\right)\right|^{2}|y|^{-1} d x d y \\
\quad \leq C \int_{I|x-t| \leq|y| \leq|I|} \sup _{\left.\left.|| R_{y}\left(e^{b}\right)(t)\right|^{-2}\left|P_{y}\left(\tilde{z}\left(e^{b}-1\right)\right)(t)\right|^{2}\right] d x \leq C\|b\|_{*}^{2} .}
\end{aligned}
$$

The first inequality is because $\left|Q_{y}\left(z^{\prime}\right)\right||y|^{-1} d x d y$ is a Carleson measure (since $\left|z^{\prime}\right| \equiv 1$ ), while the second uses $\left|R_{y}\left(e^{b}\right)\right|^{-1} \approx\left|R_{y}\left(e^{-b}\right)\right|$, John-Nirenberg, and the $L^{p}$ boundedness of the Hardy-Littlewood maximal function. The second term is dominated by $R_{y}\left(e^{b}\right)(x)^{-1} Q_{y}\left(\tilde{z}^{\prime}\left(e^{b}-1\right)\right)(x)$. This can be dealt with just as in the proof of the Carleson measure estimates for $\mu$ in Proposition 4.2.

The first and fourth terms in (4.16) are given by

$$
\bar{\partial}\left(\varphi_{y} * s(x)\right)-R_{y}\left(e^{b}\right)(x) \bar{\partial}\left(\varphi_{y} * \tau(x)\right) .
$$

Observe that

$$
\begin{aligned}
& \frac{\partial}{\partial x}\left(\varphi_{y} * s(x)\right)-R_{y}\left(e^{b}\right)(x) \frac{\partial}{\partial x}\left(\varphi_{y} * \tau(x)\right) \\
& \quad=\varphi_{y} *\left(\tilde{z}^{\prime} e^{b}\right)(x)-R_{y}\left(e^{b}\right)(x) \varphi_{y} * \tilde{z}^{\prime}(x)=0,
\end{aligned}
$$

by definition of $R_{y}$. (This is why we introduced $R_{y}$.) 
Now consider the $\partial / \partial y$ part. Because $\int(\partial / \partial y) \varphi_{y}(x) d x=0$, we can find $\alpha \in C^{\infty}$ such that $(\partial / \partial x) \alpha_{y}(x)=(\operatorname{sgn} y)(\partial / \partial y) \varphi_{y}(x)$ and supp $\alpha \subseteq[-1,1]$. Because $\varphi(x)$ is even, $\int \varphi_{y}(x) x d x=0$, so that $\int(\partial / \partial y) \varphi_{y}(x) x d x=0$, which implies that $\int \alpha=0$. Because $s^{\prime}=e^{b} \tilde{z}^{\prime}$, we obtain

$$
\begin{aligned}
& \frac{\partial}{\partial y}\left(\varphi_{y} * s(x)\right)-R_{y}\left(e^{b}\right)(x) \frac{\partial}{\partial y}\left(\varphi_{y} * \tau(x)\right) \\
& \quad=(\operatorname{sgn} y)\left[\alpha_{y} *\left(e^{b} \tilde{z}^{\prime}\right)(x)-R_{y}\left(e^{b}\right)(x)\left(\alpha_{y} * \tilde{z}^{\prime}\right)(x)\right] .
\end{aligned}
$$

Let $F(x, y)$ denote the right side of (4.19). Then

$$
|F(x, y)| \leq C\left|R_{y}\left(e^{b}\right)(x)\right|\|b\|_{*} \quad \text { if }\|b\|_{*} \text { is small enough. }
$$

Let us check this. Fix $x$ and $y$. We may suppose $R_{y}(b)(x)=0$, so that $\left|R_{y}\left(e^{b}\right)(x)\right| \geq$ $C\left|\exp \left(R_{y}(b)(x)\right)\right| \geq C$. Also, we can replace $e^{b}$ in (4.19) by $e^{b}-1$. With these normalizations, each of the two terms in (4.19) can be dominated by $\|b\|_{*}$ using (4.4), with $\varphi_{y} * f$ replaced by $R_{y} f$.

We also have that $\left|\left(R_{y}\left(e^{b}\right)(x)\right)^{-1} F(x, y)\right|^{2}|y|^{-1} d x d y$ is a Carleson measure if $\|b\|_{*}$ is small enough. Indeed, let $I$ be any interval, and let $\hat{I}=\{z \in \mathbf{C}: x \in$ $I,|y| \leq|I|\}$, and let us show that

$$
\iint_{\hat{I}}\left|\left(R_{y}\left(e^{b}\right)(x)\right)^{-1} F(x, y)\right|^{2}|y|^{-1} d x d y \leq C\|b\|_{*}^{2}|I| .
$$

We may suppose that $\int_{5 I} b=0$, and we can replace $e^{b}$ by $e^{b}-1$ in both terms in (4.19). Now those two terms can be estimated separately, using $\left|R_{y}\left(e^{b}\right)\right|^{-1} \approx$ $\left|\exp \left(-R_{y}(b)\right)\right| \approx\left|R_{y}\left(e^{-b}\right)\right|$ and arguments like those used to prove the Carleson measure estimates for $\bar{\partial}\left(R_{y}\left(e^{b}\right)(x)\right)$ before. (The first term in (4.19) is like the second term in (4.15), and vice versa.)

Altogether, $\bar{\partial} \sigma(z)=R_{y}\left(e^{b}\right)(x) \bar{\partial} \tau(z)+$ remainder, where

$$
\mid \text { remainder }|\leq C| R_{y}\left(e^{b}\right)(x) \mid\|b\|_{*}
$$

and

$$
\| \mid\left(R_{y}\left(e^{b}\right)(x)\right)^{-1} \text { (remainder) }\left.\right|^{2}|y|^{-1} d x d y\left\|_{C M} \leq C\right\| b \|_{*}^{2}
$$

if $\|b\|_{*}$ is small enough. This still holds with $\bar{\partial}$ replaced by $\partial$; therefore, since $C^{-1} \leq|\partial \tau| \leq C$,

$$
\begin{aligned}
\frac{\bar{\partial} \sigma}{\partial \sigma} & =\frac{\bar{\partial} \tau}{\partial \tau}+\text { leftovers, } \\
& \text { where } \mid \text { leftovers } \mid \leq C\|b\|_{*} \text { and } \| \mid \text { leftovers }\left.\right|^{2}|y|^{-1} d x d y\left\|_{C M} \leq C\right\| b \|_{*}^{2} .
\end{aligned}
$$

In particular, $\|\bar{\partial} \sigma / \partial \sigma\|_{\infty} \leq \frac{1}{2}\left(1+\|\bar{\partial} \tau / \partial \tau\|_{\infty}\right)<1$ if $\|b\|_{*}$ is sufficiently small.

To show that $\sigma$ is quasiconformal we must show that it is a homeomorphism on C. Assume first that $b$ is smooth and compactly supported, in addition to $\|b\|_{*}$ small. When $y \neq 0, \sigma$ is smooth (because $\tau$ is) and $\partial \sigma \neq 0$ (by the above estimates on "remainder"), and so $\sigma$ is locally a homeomorphism and also an open mapping on $\mathbf{C} \backslash \mathbf{R}$, by the inverse function theorem. This breaks down when $y=0$ because $\tau$ is not $C^{1}$ on $\mathbf{R}$, but we can repair the argument.

Fix $x_{0} \in \mathbf{R}$. Using the smoothness of $b$ and the preceding calculations of $\bar{\partial} \sigma$ and $\partial \sigma$ one can show that the Lipschitz norm of the restriction of $\sigma(z)-e^{b\left(x_{0}\right)} \tau(z)$ 
to $\left|z-x_{0}\right| \leq \delta$ tends to 0 as $\delta \rightarrow 0$. Because $\tau$ is a bilipschitz homeomorphism of $\mathbf{C}$ onto $\mathbf{C}$, this implies that if $\delta_{0}$ is small enough, then $\sigma$ maps $\left|z-x_{0}\right|<\delta_{0}$ homeomorphically onto an open set. (The proof of this is similar to the proof of the inverse function theorem.) Thus $\sigma$ is an open mapping and is locally a homeomorphism at each point in $\mathbf{C}$.

Because supp $b$ is compact, one can show that $\sigma$ tends to $\infty$ at $\infty: R_{y}\left(e^{b}\right)(x) \tau(z)$ is the main term, while the other two combine to become much smaller. (To check this, use the fact that $e^{b}-1$ has compact support.) Standard monodromy arguments imply that $\sigma$ is a homeomorphism of $\mathbf{C}$ onto itself, and is hence quasiconformal.

In the general case one approximates $b$ by $b_{j} \in C^{\infty}$ with compact support, $\left\|b_{j}\right\|_{*} \leq C\|b\|_{*}$, and $\int_{0}^{1} b_{j}(x) d x=\int_{0}^{1} b(x) d x$, as in the proof of Proposition 4.2. There is a complication now, that $R_{y}\left(e^{b_{j}}\right)(x)$ converges uniformly on compact subsets of $\mathbf{C} \backslash \mathbf{R}$, but not of $\mathbf{C}$ itself. However, the normalizations force that for any compact subset $K$ of $\mathbf{C},\left|R_{y} b_{j}(x)\right| \leq C_{1}\left\|b_{j}\right\|_{*}|\log y|+C_{2}(K)$ for $x+i y \in K$. Here $C_{1}$ does not depend on $K$, but $C_{2}$ does, because there is also logarithmic growth in $x$. If $\|b\|_{*}$ is small enough, then

$$
\left|R_{y}\left(e^{b_{j}}\right)(x)\right| \leq C\left|\exp \left(R_{y} b_{j}(x)\right)\right| \leq C(K)|y|^{-1 / 2}
$$

for $x+i y \in K$. In (4.12), though, this gets hit by $\left|\tau(z)-\varphi_{y} * \tau(x)\right| \leq C|y|$. From this one obtains that $\sigma_{j} \rightarrow \sigma$ uniformly on compact subsets of $\mathbf{C}$ if we also make the normalization $s_{j}(0)=s(0)$ for all $j$. Thus $\sigma$ must be quasiconformal if $\|b\|_{*}$ is small enough.

Therefore $\rho=\sigma \circ \tau^{-1}$ must be q.c., and its dilatation $\mu$ satisfies $\|\mu\|_{\infty} \leq C\|b\|_{*}$ and $|\mu|^{2} \delta_{\Gamma}(z)^{-1} d x d y$ is a Carleson measure relative to $\Gamma$ with norm $\leq C\|b\|_{*}^{2}$. This follows from the well-known formula for the dilatation of a composition (see [A2]),

$$
\mu_{h \circ f^{-1}} \circ f=\frac{\partial f}{\bar{\partial} \bar{f}} \frac{\mu_{h}-\mu_{f}}{1-\bar{\mu}_{f} \mu_{h}},
$$

and the estimates $(4.20)$.

The estimates for the higher gradients of $\mu$ can be obtained similarly, using also the estimates for the higher derivatives of $\tau$ in Lemma 4.11. This completes the proof of Proposition 4.13.

5. A problem in conformal welding. Let $\Gamma$ be a Jordan curve that passes through $\infty$, let $\Omega_{+}$and $\Omega_{-}$denote its complementary regions, and let $\Phi_{+}$and $\Phi_{-}$ be conformal maps of the UHP and LHP onto $\Omega_{+}$and $\Omega_{-}$such that $\Phi_{ \pm}(\infty)=\infty$. Define a homeomorphism $h$ on $\mathbf{R}$ by $h=\left(\left.\Phi_{-}\right|_{\Gamma}\right)^{-1} \circ\left(\left.\Phi_{+}\right|_{\mathbf{R}}\right)$. The problem is to go back and forth between $\Gamma$ and $h$. Note that $h$ controls the relationship of the harmonic measure on the two sides of $\Gamma$.

If $\Gamma$ is a quasicircle, then $h$ satisfies the doubling condition

$$
M^{-1} \leq \frac{h(x+t)-h(x)}{h(x)-h(x-t)} \leq M .
$$

Conversely, to every such $h$ there corresponds a Jordan curve $\Gamma$, unique up to affine transformations on $C$, and $\Gamma$ is a quasicircle. See [A1]

The problem is to characterize the welding homeomorphisms that correspond to the class of chord-arc curves, or, more generally, to rectifiable curves. From Lavrentiev's theorem and basic properties of $A_{\infty}$ weights it follows that $h^{\prime} \in A_{\infty}$ 
if $\Gamma$ is chord-arc. (Of course, $h$ is locally absolutely continuous if $\Gamma$ is locally rectifiable.) David [D2] showed that chord-arc curves with small constant correspond precisely to those $h^{\prime}$ s such that $\left\|\log h^{\prime}\right\|_{*}$ is small. Unfortunately, $h^{\prime} \in A_{\infty}$ does not characterize chord-arc curves: There is a nonrectifiable quasicircle such that $C^{-1} \leq h^{\prime} \leq C$ (see [S1]). However, Bishop, Carleson, Garnett, and Jones [BCGJ] have characterized the curves such that $h$ is absolutely continuous when $\Gamma$ is a bounded Jordan curve; $\Gamma$ must be nearly rectifiable in a certain precise sense.

In this section we consider David's result. One direction, that if $\Gamma$ is chordarc with small constant then $\left\|\log h^{\prime}\right\|_{*}$ is small, follows from conformal mapping estimates due to Lavrentiev and Pommerenke. (See also the next section.)

Conversely suppose $\left\|\log h^{\prime}\right\|_{*}$ is small, so that $h^{\prime} \in A_{\infty}$ and (5.1) holds. Ahlfors [A1] gets the curve from $h$ as follows. First extend $h$ to a (sense-reversing) q.c. map of the UHP to the LHP. Then $g^{*}(z)=g(\bar{z})$ is sense preserving and takes the LHP to itself. Let $\mu$ be its dilatation, and set $\mu=0$ in the UHP. Let $\rho$ be a q.c. map on $C$ with dilatation $\mu$. As in $[\mathbf{A} 1], \Gamma=\rho(\mathbf{R})$ has $h$ as a welding homeomorphism. From Proposition 4.2 and Theorem 0.1 it follows that $\Gamma$ is a chord-arc curve with small constant if $\left\|\log h^{\prime}\right\|_{*}$ is small.

6. The Riemann mapping for chord-arc curves and the theorem of Coifman and Meyer. In this section we give a new approach to Coifman and Meyer's theorem [CM3] on the real analyticity of the Riemann mapping as a function of the chord-arc curve. Let us first recall what all this means. (See also [S2].)

Let $\Gamma_{0}$ be a fixed oriented chord-arc curve with arclength parameterization $z_{0}(t)$. If $b \in \mathrm{BMO}(\mathbf{R})$ is real valued and $\|b\|_{*}$ is small enough, then

$$
z(t)=z_{0}(0)+\int_{0}^{t} e^{i b(s)} z_{0}^{\prime}(s) d s
$$

is an arclength parameterization of another chord-arc curve $\Gamma$. This gives a natural notion of a small neighborhood of $\Gamma_{0}$. Using this one can turn the space of all chord-arc curves into a Banach manifold (modeled on BMO) in a natural way. In fact, David [D1] has shown that by defining $\arg z^{\prime}(t)$ carefully this space can be identified with an open subset of real-valued $\mathrm{BMO}(\mathbf{R})$.

Let $\Phi$ denote a conformal map of the UHP onto the left side of $\Gamma$ such that $\Phi(\infty)=\infty$. Define a homeomorphism $h: \mathbf{R} \rightarrow \mathbf{R}$ by $h(t)=\Phi^{-1}(z(t))$. From Lavrentiev's theorem it follows that $h^{\prime} \in A_{\infty}$, and hence $\log h^{\prime} \in$ BMO. Observe that if $\tilde{\Phi}$ is another such conformal mapping, then $\tilde{\Phi}(z)=\Phi(c z+d)$ for some $c>0$ and $d \in \mathbf{R}$, so that $\log \tilde{h}^{\prime}=\log h^{\prime}+\log c$. Therefore, as elements of BMO, $\log \tilde{h}^{\prime}$ and $\log h^{\prime}$ are the same, and $\log h^{\prime}$ does not depend on the choice of $\Phi$.

The theorem of Coifman and Meyer is that for each $\Gamma_{0}, z_{0}(t)$, the correspondence $b \mapsto \log h^{\prime}$ is a real analytic mapping from a neighborhood of $0 \in$ BMO into $\mathrm{BMO}$. This means that this function has a norm convergent multilinear series, with estimates on the terms; see [CM2, 3] (or [S2]) for a precise definition. In particular, the mapping has Frechet derivatives of all orders, and if $\|b\|_{*}$ is small enough, then $\left\|\log h^{\prime}-\log h_{0}^{\prime}\right\|_{*} \leq C\|b\|_{*}$.

It is very important to use $h$, which is defined in terms of $\Phi^{-1}$, rather than $\Phi$ itself. For one thing, as an element of BMO, $\log \Phi^{\prime}$ is not independent of the choice of $\Phi$. Also, even though $h^{\prime} \in A_{\infty}$ implies $\left(h^{-1}\right)^{\prime} \in A_{\infty}$, the correspondence 
$h \mapsto h^{-1}$ is not even continuous in the BMO topology, except at the origin (i.e., about the identity map).

Even the continuity of the mapping $b \mapsto \log h^{\prime}$ in the BMO topology was not known until the theorem of Coifman and Meyer, except when $\Gamma_{0}=\mathbf{R}$. In that case a small neighborhood of $\Gamma_{0}$ consists of the chord-arc curves with small constant [CM2]. Since $h_{0}(x) \equiv x$ if $\Gamma_{0}=\mathbf{R}$, the continuity of $b \mapsto \log h^{\prime}$ about $\Gamma_{0}$ is just the statement that $\left\|\log h^{\prime}\right\|_{*}$ is small if the chord-arc constant of $\Gamma$ is. This is equivalent to $\left\|\log \Phi^{\prime}\right\|_{*}$ being small, which had been proved earlier (see [P1, 2, 3]). (This is the conformal mapping estimate mentioned in §5.) The simpler special case of $\Gamma_{0}=\mathbf{R}$ should be kept in mind throughout this section.

Let us show how to apply the methods of this paper to the mapping $b \mapsto \log h^{\prime}$. Define $r_{b}=z \circ z_{0}^{-1}$ and $\beta=b \circ z_{0}^{-1}$, so that

$$
r_{b}(w)=r_{b}\left(w_{0}\right)+\int_{w_{0}}^{w} e^{i \beta(z)} d z, \quad w_{0}, w \in \Gamma_{0},
$$

where the integral is taken over the corresponding arc on $\Gamma_{0}$. By Proposition 4.13 (or 4.2 when $\Gamma=\mathbf{R}_{0}$ ) there is a q.c. extension $\rho_{b}$ of $r_{b}$ given by (4.12) ((4.1) when $\left.\Gamma_{0}=\mathbf{R}\right)$ whose dilatation $\mu_{b}$ lies in $M\left(\Gamma_{0}\right) \cap N\left(\Gamma_{0}\right)$ with norm $\leq C\|b\|_{*}$.

The mapping $b \mapsto \mu_{b}$ is real analytic. One can easily write down a formal power series expansion, but the issue is whether one can get uniform norm estimates on the terms of the power series. By $\S 4$, the mappings $b \mapsto \rho_{b} \mapsto \mu_{b}$ are perfectly alright if $b$ is complex-valued, as long as $\|b\|_{*}$ is small. In particular one gets the same estimates for $\mu_{b}$. This complex extension is also formally complex analytic. Moreover, using Cauchy's theorem like in $\S 10$ of [CM2], one can estimate the terms of the power series for $\mu_{b}$ in $b$ in terms of the boundedness of $\left\|\mu_{b}\right\|_{M \cap N}$. This gives the real analyticity of $b \mapsto \mu_{b}$.

Let us show how to go from $\mu_{b}$ to $h$. We use a well-known set-up from q.c. mappings (see e.g. [A3]) together with our estimates. Suppose first that $\Gamma_{0}=\mathbf{R}$. Consider the restriction of $\rho_{b}$ to the UHP, a q.c. map onto the left side of $\Gamma$. Then $\rho_{b} \circ \eta^{-1}$ is conformal if $\eta$ is a q.c. map of the UHP onto itself with dilatation $\mu_{b}$ there. Define $\nu=\nu_{b}$ by $\nu=\mu_{b}$ on the UHP and $\nu(z)=\overline{\nu(\bar{z})}$ on the LHP, and let $\eta: \mathbf{C} \rightarrow \mathbf{C}$ be the q.c. map with dilatation $\nu$ satisfying $\eta(0)=0, \eta(1)=1$. Then $\eta$ maps the UHP to itself, and $\rho_{b} \circ \eta^{-1}$ is conformal. Because $\rho_{b}(t)=z(t), t \in \mathbf{R}$, we get that $\left.\eta\right|_{\mathbf{R}}=h$. From Theorem 0.1 it follows that $\left\|\log h^{\prime}\right\|_{*} \leq C\left\|\mu_{b}\right\|_{M+N} \leq C\|b\|_{*}$ if $\|b\|_{*}$ is small enough.

Furthermore, in the notation of $\S 2, \mu_{b} \in 2$ - GMN(R). This follows from the Carleson measure estimates on $\nabla^{j} \mu, j=0,1,2$, and Sobolev's lemma. Thus the method of $\S 2$ is applicable. This is good because that method gives an explicit power series for $\log \eta_{z}$ in terms of $\nu$, which is exactly what we want.

There is a disgusting technical point here, which is that we made some a priori assumptions in $\S 2$. Morally, it is the estimates that are important, and we shall not worry now about the issue of getting rid of the a priori assumptions. However, one way to do this is indicated below.

Consider the case of general $\Gamma_{0}$. Let $\Phi_{0}$ and $h_{0}$ be as before. Then $\rho_{b} \circ \Phi_{0}$ defines a q.c. map of the UHP onto the left side of $\Gamma$, with dilatation equal to $\left(\mu_{b} \circ \Phi_{0}\right) \overline{\Phi_{0}^{\prime}} / \Phi_{0}^{\prime}$. (See the formula at the end of $\S 4$.) Set $\nu$ equal to this on the 
UHP, define $\nu(z)=\overline{\nu(\bar{z})}$ on the LHP, and let $\eta$ be as before. Then $\Phi=\rho_{b} \circ \Phi_{0} \circ \eta^{-1}$ is conformal, and $h=\left(\left.\eta\right|_{\mathbf{R}}\right) \circ h_{0}$, because $\left.\rho_{b}\right|_{\Gamma_{0}}=r_{b}=z \circ z_{0}^{-1}$.

We need to control $\nu$. Because $\log \Phi_{0}^{\prime} \in$ BMOA (see $[\mathbf{J K}]$ ), we have that

$$
\left|y^{n} \nabla^{n}\left\{\overline{\Phi_{0}^{\prime}}\left(\Phi_{0}^{\prime}\right)^{-1}\right\}\right|^{2} y^{-1} d x d y
$$

is a Carleson measure if $n \geq 1$. For $\mu_{b} \circ \Phi_{0}$, first observe that if $|\alpha(z)| d x d y$ is a Carleson measure on $\Omega_{+}^{0}=$ the left side of $\Gamma_{0}$, then $\left|\alpha\left(\Phi_{0}(z)\right) \Phi_{0}^{\prime}(z)\right| d x d y$ is a Carleson measure on the UHP. This could be proved in much the same way as Lemma 4.8. (In fact, one could apply Lemma 4.8 to $\pi \circ \Phi_{0}$, where $\pi\left(\Omega_{+}^{0}\right)=$ UHP and $\pi$ is bilipschitz.) Alternatively, the bilipschitz map $\tau: \mathbf{C} \rightarrow \mathbf{C}$ such that $\tau(\mathbf{R})=\Gamma_{0}$ given by Lemma 4.11 is given on the UHP by $\Phi_{+} \circ \rho_{+}$, where in particular $\rho_{+}^{-1}$ satisfies Lemma 4.8. From this one gets the desired property of $\Phi_{0}$, because $\tau$ is bilipschitz.

From $\mu_{b} \in N\left(\Gamma_{0}\right)$ one gets that $\mu_{b} \circ \Phi_{0} \in N(\mathbf{R})$. This uses the preceding remarks and the fact that $\delta_{\Gamma}\left(\Phi_{0}(z)\right) \approx\left|\Phi_{0}^{\prime}(z)\right| y$ (see, e.g., (1.6) of [JK]). Hence $\nu \in N(\mathbf{R})$. One proves similarly that $\left(y^{n}\left|\nabla^{n} \nu\right|\right)^{2} y^{-1} d x d y$ is a Carleson measure if $n>0$. In particular, $\nu \in 2-G M N(\mathbf{R})$. Thus one can apply Theorem 0.1 to get that if $g=\left.\eta\right|_{\mathbf{R}}$, then $\|g\|_{*} \leq C\|b\|_{*}$ if $\|b\|_{*}$ is small enough. From this and $h_{0}^{\prime} \in A$ we get that $\left\|\log h^{\prime}-\log h_{0}^{\prime}\right\|_{*} \leq C\|b\|_{*}$. As before, the method of $\S 2$ gives a power series for $g$ in terms of $\nu$, and hence $b$, modulo a priori assumptions.

Let us indicate another method for computing $\log h^{\prime}$ in terms of $b$ that is more in the spirit of $[\mathbf{C M 2}, 3]$.

Let $\Phi_{0}$ and $\Phi$ be as before. By the results of Lavrentiev and Pommerenke (see $[\mathbf{P 1}, 3$ or $\mathbf{J K}]$ ), $\log \Phi^{\prime}$ and $\log \Phi_{0}^{\prime}$ lie in BMOA of the UHP, and hence have boundary values almost everywhere on $\mathbf{R}$. Define $B(x)=\operatorname{Im} \log \Phi^{\prime}(h(x))$ and $B_{0}(x)=\operatorname{Im} \log \Phi_{0}^{\prime}\left(h_{0}(x)\right)$ on $R$. Because $z(t)=\Phi(h(t)), z^{\prime}(t)=e^{i B(t)}$ a.e., so that $B$ is a choice of $\arg z^{\prime}$. Let $H$ denote the Hilbert transform and define $V_{h}$ by $V_{h} f=f \circ h$. The analyticity of $\log \Phi^{\prime}$ implies that $H\left(\operatorname{Re} \log \Phi^{\prime}\right)=\operatorname{Im} \log \Phi^{\prime}$, which itself yields (as in [CM1, 2])

$$
\log h^{\prime}=V_{h} H V_{h}^{-1}(B) \text {. }
$$

Morally, it is clear that $B$ and $B_{0}+b$ should be the same when $\|b\|_{*}$ is small, but because $\arg z^{\prime}(t)$ is not well defined, we must be careful. Let us assume for now that $B=B_{0}+b$, and prove it afterwards.

Let $\nu, \eta$ be as above, so that $\left.\eta\right|_{\mathbf{R}}=g, h=g \circ h_{0}$, and $\nu$ is the dilatation of $\eta$. We can rewrite $(6.1)$ as

$$
\log h^{\prime}=V_{h_{0}} V_{g} H V_{g}^{-1} V_{h_{0}}^{-1}\left(B_{0}+b\right),
$$

since $V_{h}=V_{h_{0}} V_{g}$. Since $h_{0}^{\prime} \in A_{\infty}, V_{h_{0}}$ and $V_{h_{0}}^{-1}$ are bounded operators on BMO. Also, $V_{g} H V_{g}^{-1}(f)$ can be computed in terms of a $\bar{\partial}-\nu \partial$ problem: if $F$ is defined on $\mathbf{C} \backslash \mathbf{R}$, has jump $f$ across $\mathbf{R}$, satisfies $(\bar{\partial}-\nu \partial) F=0$ off $\mathbf{R}$, and also satisfies certain estimates, then the boundary values of $F$ on $\mathbf{R}$ from the UHP are given by $\frac{1}{2} f+(1 / 2 i) V_{g} H V_{g}^{-1}(f)$. (The case $\nu \equiv 0, g(x) \equiv x$, is the classical case.) This is shown in $\S 5$ of $[\mathbf{S 3}]$.

It is also shown in [S3] that if $\nu \in N(\mathbf{R})$ has sufficiently small norm, then $F$ as above exists with BMO boundary values if $f \in \operatorname{BMO}(\mathbf{R})$, and that $F$ is given as a power series in $\nu$, all with the right norm estimates. Since we already know that $\nu$ 
has a good power series in $b$, we conclude from (6.2) that $\log h^{\prime}$ can be expressed as a power series in $b$ (if $\|b\|_{*}$ is sufficiently small). Notice that we do not run into the earlier problems with a priori assumptions here, because none are made in [S3].

It is interesting to compare the previous argument with the method of $\S 3$. In both we use [S3] to go from estimates on $\nu$ to estimates on $V_{g} H V_{g}^{-1}$. In the preceding we went from there to $\log h^{\prime}$ using (6.2). In $\S 3$ we went to $\log g^{\prime}$ directly from the estimates for $V_{g} H V_{g}^{-1}$ and the formula for its kernel.

Let us check that $B=B_{0}+b$ if $\|b\|_{*}$ is small enough. It suffices to show that $\left\|B-B_{0}\right\|_{*}$ is small if $\|b\|_{*}$ is. For we know that $z^{\prime}(t)=e^{i B(t)}, z_{0}^{\prime}(t)=e^{i B_{0}(t)}$, and that $z^{\prime}(t)=z_{0}^{\prime}(t) e^{i b(t)}$. Thus $e^{i\left(b-B_{0}-b\right)}=1$ a.e., so that $(1 / 2 \pi)\left(B-B_{0}-b\right)$ is integer valued. If $\|b\|_{*}$ is small, then so is $\left\|B-B_{0}-b\right\|_{*}$; if $(1 / 2 \pi)\left(B-B_{0}-b\right)$ is also integer valued, it must be constant, i.e., $=0$ as an element of BMO.

To show that $\left\|B-B_{0}\right\|_{*}$ is small if $\|b\|_{*}$ is, we use (6.1). Our earlier arguments (i.e., applying Theorem 0.1 to $\eta$ ) show that $g=\left.\eta\right|_{\mathbf{R}}$ satisfies $\left\|\log g^{\prime}\right\|_{*} \leq$ $C\|\nu\|_{M+N} \leq C\|b\|_{*}$ if $\|b\|_{*}$ is small enough. Since $h_{0}^{\prime} \in A_{\infty},\left\|\log h^{\prime}-\log h_{0}^{\prime}\right\|_{*}=$ $\left\|\log g^{\prime} \circ h_{0}\right\|_{*} \leq C\left\|\log g^{\prime}\right\|_{*}$. By (6.1),

$$
B_{0}=-V_{h_{0}} H V_{h_{0}}^{-1}\left(\log h_{0}^{\prime}\right) \quad \text { and } \quad B=-V_{h} H V_{h}^{-1}\left(\log h^{\prime}\right)
$$

Hence

$$
B-B_{0}=-V_{h} H V_{h}^{-1}\left(\log h^{\prime}-\log h_{0}^{\prime}\right)-\left(V_{h} H V_{h}^{-1}-V_{h_{0}} H V_{h_{0}}^{-1}\right)\left(\log h_{0}^{\prime}\right) .
$$

Because $h_{0}^{\prime} \in A_{\infty}$, we have $h^{\prime} \in A_{\infty}$ with uniform estimates on the $A_{\infty}$ constants if $\left\|\log h^{\prime}-\log h_{0}^{\prime}\right\|_{*} \leq C\|b\|_{*}$ is small enough, so that

$$
\left\|V_{n} H V_{n}^{-1}\left(\log h^{\prime}-\log h_{0}^{\prime}\right)\right\|_{*} \leq C\|b\|_{*} .
$$

Also,

$$
V_{h} H V_{h}^{-1}-V_{h_{0}} H V_{h_{0}}^{-1}=V_{h_{0}}\left(V_{g} H V_{g}^{-1}-H\right) V_{h_{0}}^{-1}
$$

has operator norm $\leq C\|\nu\|_{N}$ on BMO if $\|\nu\|_{N}$ is small enough; as before, this follows from $\S 5$ of $[\mathbf{S 3}]$. Since $\log h_{0}^{\prime} \in \mathrm{BMO}$, we get that $\left\|B-B_{0}\right\|_{*} \leq C\|b\|_{*}$ if $\|b\|_{*}$ is small enough, as desired.

\section{REFERENCES}

[A1] L. V. Ahlfors, Quasiconformal reflections, Acta Math. 109 (1963), 291-301.

[A2] _ L Lectures on quasiconformal mappings, Van Nostrand, New York, 1966.

[A3] _ Conformality with respect to Riemannian metrics, Ann. Acad. Sci. Fenn. Ser. A1 206 (1955), 3-22.

[AB] L. V. Ahlfors and L. Bers, Riemann's mapping theorem for variable metrics, Ann. of Math. (2) 72 (1960), 385-404.

[BCGJ] C. Bishop, L. Carleson, J. Garnett, and P. Jones, Harmonic measure supported on curves, preprint.

[C] L. Carleson, On mappings conformal at the boundary, J. Analyse Math. 19 (1967), 1-13.

[CF] R. Coifman and C. Fefferman, Weighted norm inequalities for maximal functions and singular integrals, Studia Math. 51 (1974), 241-250.

[CM1] R. Coifman and Y. Meyer, Le théorème de Calderón par les méthods de variable reele, $\mathrm{C} . \mathrm{R}$. Acad. Sci. Paris Sér. A 289 (1979), 425-428.

[CM2] _ Une généralisation du théorème de Calderón sur l'intégrale de Cauchy, Fourier Analysis (Proc. Conf., El Escorial, Spain, 1979) (M. de Guzmán and I. Peral, eds.), 1980.

[CM3] _ Lavrentiev's curves and conformal mapping, Institut Mittag-Leffler, Report No. 5, 1983. 
[CDM] R. Coifman, G. David, and Y. Meyer, La solution des conjectures de Calderón, Adv. in Math. 48 (1983), 144-148.

[CMS] R. Coifman, Y. Meyer, and E. Stein, Some new function spaces and their applications to analysis, J. Funct. Anal. 62 (1985), 304-335.

[D1] G. David, Thèse de troisième cycle, Université de Paris XI, Orsay, France

[D2] _ Courbes corde-arc et espaces de Hardy généralises, Ann. Inst. Fourier (Grenoble) 32 (1982), 227-239.

[D3] _ - Opérateurs intégraux singulièrs sur certaines courbes du plan complex, Ann. Sci. École Norm. Sup. 17 (1984), 157-189.

[DJS] G. David, J. L. Journé, and S. Semmes, A generalized boundedness criterion for singular integral operators, Rev. Mat. Iberoamericana 1 (1985), 1-56.

[Dh] B. E. J. Dahlberg, On the absolute continuity of elliptic measures, preprint.

[FS] C. Fefferman and E. Stein, $H^{p}$ spaces of several variables, Acta Math. 129 (1972), 137-193.

[G] J. B. Garnett, Bounded analytic functions, Academic Press, 1981.

[Je] J. L. Journé, Calderón-Zygmund operators, pseudo-differential operators, and the Cauchy integral of Calderón, Lecture Notes in Math., vol. 994, Springer-Verlag, Berlin and New York, 1983.

[J8] P. Jones, Homeomorphisms of the line that preserve BMO, Ark. Mat. 21 (1983), 229-231.

[JK] D. Jerison and C. Kenig, Hardy spaces, $A_{\infty}$, and singular integrals on chord-arc domains, Math. Scand. 50 (1982), 221-247.

[LV] O. Lehto and K. Virtanen, Quasiconformal mappings in the plane, Springer-Verlag, 1973.

[P1] Chr. Pommerenke, Schlichte Funktion und analytische Funktionen von beschrankter mittlerer Oszillation, Comment. Math. Helv. 52 (1977), 591-602.

[P2] _ - On univalent functions, Bloch functions, and VMOA, Math. Ann. 236 (1978), 199-208.

[P3] _ Boundary behavior of conformal mappings, Aspects of Contemporary Complex Analysis (D. Brannan and J. Clunie, eds.), Academic Press, 1980.

[S1] S. Semmes, A counterexample in conformal welding concerning chord-arc curves, Ark. Mat. 24 (1986), 141-158.

[S2] - The Cauchy integral, chord-arc curves, and quasiconformal mappings (Proc. Bieberbach Conf., Purdue Univ., 1985) (Baernstein, Drasin, Durin and Marden, eds.), Math. Surreys, no. 21, Amer. Math. Soc., Providence, R.I., 1986.

[S3] _ Estimates for $(\bar{\partial}-\mu \partial)^{-1}$ and Calderón's theorem on the Cauchy integral, Trans. Amer. Math. Soc. 306 (1988), 191-232.

[St] E. M. Stein, Singular integrals and differentiability properties of functions, Princeton Univ. Press, Princeton, N. J., 1970.

[Str] J. O. Stromberg, Bounded mean oscillation with Orlicz norms and duality of Hardy spaces, Bull. Amer. Math. Soc. 82 (1976), 953-955.

[Tu1] P. Tukia, The planar Schönflies theorem for Lipschitz maps, Ann. Acad. Math. Sci. Fenn. Ser. A I Math. 5 (1980), 49-72.

[Tu2] _ Extension of quasisymmetric and Lipschitz embeddings of the real line into the plane, Ann. Acad. Math. Sci. Fenn. Ser. A I iMath. 86 (1981), 89-94.

[V] N. Varopoulos, BMO functions and the $\bar{\partial}$ equation, Pacific J. Math. 74 (1977), 257-259.

Department of Mathematics, Yale University, New haven, Connecticut 06520

Department of Mathematics, Rice University, Houston, TeXas 77251 